\title{
Effective supersymmetry at the LHC
}

\author{
Howard Baer, ${ }^{a}$ Sabine Kraml, ${ }^{b}$ Andre Lessa, ${ }^{a}$ Sezen Sekmen ${ }^{c}$ and Xerxes Tata ${ }^{d, e}$ \\ ${ }^{a}$ Dept. of Physics and Astronomy, University of Oklahoma, \\ 440 West Brooks, Norman, OK 73019, U.S.A. \\ ${ }^{b}$ Laboratoire de Physique Subatomique et de Cosmologie, UJF Grenoble 1, CNRS/IN2P3, INPG, \\ 53 Avenue des Martyrs, F-38026 Grenoble, France \\ ${ }^{c}$ Dept. of Physics, Florida State University, \\ 315 Keen Bldg, Tallahassee, FL 32306, U.S.A. \\ ${ }^{d}$ Dept. of Physics and Astronomy, University of Hawaii, \\ 2505 Correa Road, Honolulu, HI 96822,U.S.A. \\ ${ }^{e}$ Dept. of Physics, University of Wisconsin, \\ 1150 University Ave., Madison, WI 53706, U.S.A. \\ E-mail: baer@nhn.ou.edu, sabine.kraml@lpsc.in2p3.fr, lessa@nhn.ou.edu, \\ sezen.sekmen@cern.ch, tata@phys.hawaii.edu
}

ABSTRACT: We investigate the phenomenology of Effective Supersymmetry (ESUSY) models wherein electroweak gauginos and third generation scalars have masses up to about $1 \mathrm{TeV}$ while first and second generation scalars lie in the multi-TeV range. Such models ameliorate the SUSY flavor and CP problems via a decoupling solution, while at the same time maintaining naturalness. In our analysis, we assume independent GUT scale mass parameters for third and first/second generation scalars and for the Higgs scalars, in addition to $m_{1 / 2}, \tan \beta$ and $A_{0}$, and require radiative electroweak symmetry breaking as usual. We analyse the parameter space which is consistent with current constraints, by means of a Markov Chain Monte Carlo scan. The lightest MSSM particle (LMP) is mostly, but not always the lightest neutralino, and moreover, the thermal relic density of the neutralino LMP is frequently very large. These models may phenomenologically be perfectly viable if the LMP before nucleosynthesis decays into the axino plus SM particles. Dark matter is then an axion/axino mixture. At the LHC, the most important production mechanisms are gluino production (for $m_{1 / 2} \lesssim 700 \mathrm{GeV}$ ) and third generation squark production, while SUSY events rich in $b$-jets are the hallmark of the ESUSY scenario. We present a set of ESUSY benchmark points with characteristic features and discuss their LHC phenomenology.

KeYwords: Supersymmetry Phenomenology

ARXIV EPRINT: 1007.3897 


\section{Contents}

1 Introduction $\quad 1$

2 Viable spectra and parameter space of the ESUSY model 5

3 Markov chain Monte Carlo analysis of ESUSY 9

3.1 Setup of the MCMC scans 9

3.2 MCMC results with neutralino LMP 11

$\begin{array}{lll}3.3 & \text { Effective SUSY benchmark points } & 13\end{array}$

4 Phenomenology of effective SUSY at the LHC $\quad \mathbf{1 5}$

4.1 Light gluinos with $\widetilde{Z}_{1}$ as LMP (ES1) 17

4.2 Light top and bottom squarks, heavy gluinos and $\widetilde{Z}_{1}$ as LMP (ES2 \& ES3) 19

4.3 Bottom or top squarks as LMP: the case of heavy quasi-stable colored particles (ES4) 20

4.4 Staus as LMP: the case of heavy quasi-stable charged particles (ES5) 20

$\begin{array}{llr}5 & \text { Summary and conclusions } & 21\end{array}$

\section{Introduction}

Particle physics models that include weak scale softly broken supersymmetry (SUSY) are especially compelling in that they stabilize the weak scale, even in the presence of new physics at much higher energy scales, such as $M_{\text {Planck }}$ or $M_{\text {GUT }}$. The simplest of such models, the Minimal Supersymmetric Standard Model (MSSM) [1, 2] enjoys some compelling indirect experimental support in that the measured values of the gauge couplings at energy scale $Q=M_{Z}$, when run to high energies via renormalization group evolution (RGE), nearly unify at $Q=M_{\mathrm{GUT}} \simeq 2 \times 10^{16} \mathrm{GeV}$, as is expected in the simplest grand unified theories (GUTs). On the astrophysical side, SUSY theories with a conserved $R$-parity offer several candidates for the dark matter particle, among these: the lightest neutralino, the gravitino and the axion/axino supermultiplet.

Along with these successes, generic SUSY models also lead to new puzzles not present in the Standard Model (SM). Additional (usually discrete) symmetries are necessary to prevent a too rapid rate for proton decay. Moreover, unless weak scale soft SUSY breaking (SSB) parameters of the MSSM are flavor-blind [1] or "aligned" [3], and nearly real, the model leads to unacceptably large rates for flavor-changing transitions and $C P$ violating effects. We stress that various (sometimes, admittedly ad hoc) mechanisms have been suggested to ameliorate these undesired effects which, we speculate, arise because of our lack of understanding of how SM superpartners feel the effects of SUSY breaking. 
Another possibility to suppress unwanted flavor-changing and $C P$-violating effects is to arrange for matter sparticles to be heavy so that their effects are sufficiently suppressed [4]. Large matter scalar masses also serve to suppress proton decay processes in a SUSY GUT. This decoupling solution to the SUSY flavor problem usually requires SSB terms of order 10-100 TeV [5], well beyond expectations from naturalness, which favors weak scale soft terms. It was noted as far back as 1986 [6], and later again in ref. [7, 8], that the stability of the weak scale to radiative corrections requires only the electroweak (EW) gauginos and third generation sparticles - these couple with large strength to the Higgs sector - to have masses up to $\mathcal{O}(1) \mathrm{TeV}$, while gluinos and superpartners of the first two generations (whose direct couplings to the Higgs sector are very small, so that these enter naturalness considerations only at the two-loop level) could well have multi-TeV masses. Since the most stringent constraints on flavour- and $C P$-violation come from the first two generations of quarks and leptons, such a mass spectrum potentially alleviates the SUSY flavor and $C P$ problems without the need for undue fine-tuning of parameters. This was subsequently developed into the framework referred to as "Effective Supersymmetry" (ESUSY), by Cohen, Kaplan and Nelson [9], who suggested two different realizations of the split matter generations idea by introducing a SUSY-breaking sector to which the first two generations couple more strongly than the third generation. As a result, the matter scalars of the first two generations acquire larger SUSY-breaking masses than third generation scalars.

The ESUSY scenario seems in recent years to have become less favored due to two measurements. The first - the measured $(2-3) \sigma$ deviation in $(g-2)_{\mu}$ from its Standard Model (SM) expectation $[10]^{1}$ — seems to require smuons/muon sneutrinos in the sub$\mathrm{TeV}$ range if the deviation is to be attributed to SUSY. The second - the increasingly precise measurement of the dark matter (DM) density of the Universe - is difficult to reconcile in ESUSY if the dark matter is assumed to be dominantly composed of thermal relic neutralinos left over in standard Big Bang cosmology. With scalars in the multi$\mathrm{TeV}$ range, along with a bino-like neutralino, the relic density is calculated to be typically several orders of magnitude higher than the experimentally observed value [11, 12],

$$
\Omega_{\mathrm{DM}} h^{2}=0.1123 \pm 0.0035,
$$

although there are some special parameter regions where this need not be the case.

At the present time, it is not completely clear whether the $(g-2)_{\mu}$ anomaly is real. The current discrepancy arises if one adopts the (more direct to use) hadronic vacuum polarization amplitude from low energy $e^{+} e^{-} \rightarrow$ hadrons data. If instead the vacuum polarization is taken from $\tau$ lepton decay data, then the discrepancy is smaller (but recently growing!).

In the case of neutralino DM, it is possible to have a small superpotential $\mu$ term coexisting with large scale masses, as occurs e.g. in the hyperbolic branch/focus point region of the mSUGRA ${ }^{2}$ model. Then the lightest neutralino, instead of being typically binolike with a small annihilation cross section and concomitantly large relic density, becomes mixed higgsino dark matter with thermal relic neutralinos from the Big Bang making up

\footnotetext{
${ }^{1}$ Update on $(g-2)_{\mu}$

${ }^{2}$ Minimal Supersymmetric model with Universal soft terms at the Gut scale and RAdiative electroweak symmetry breaking.
} 
the observed cold dark matter relic density. Other possibilities for obtaining the right relic density are resonant annihilation through the pseudoscalar Higgs, or co-annihilation with third-generation sfermions.

Regions of parameter space where the neutralino relic density is too large cannot be unequivocally excluded in extensions of the model. For instance, if one invokes the PecceiQuinn-Weinberg-Wilczek (PQWW) solution to the strong CP problem [13-16], then one expects the presence of an axion/axino supermultiplet in SUSY theories. If the axino $\tilde{a}$ is the lightest SUSY particle (LSP), then $\widetilde{Z}_{1} \rightarrow \tilde{a} \gamma$ and other decay modes are allowed, which can greatly reduce the DM abundance far below the level expected from neutralinos. The scenario of mixed axion/axino DM has been examined in the SUGRA context recently in ref. [17-19] in a general 19 parameter MSSM, and appears to be at least as viable as the case with neutralino DM.

In light of these considerations, we feel it would be fruitful to re-visit some of the phenomenological implications of ESUSY models at the beginning of the LHC era. In our analysis, we subsume the qualitative features of the ESUSY model: i.e. third generation, Higgs sector and EW gaugino masses at or below the TeV scale, with multi-TeV SSB parameters for the first two generations. For simplicity we also assume gaugino mass unification. ${ }^{3}$ While we adopt the qualitative picture of Effective SUSY, we do not assume the validity of the more speculative mechanisms that the authors of ref. [9] suggest for the hierarchy between the SSB parameters of the first two generations and the corresponding parameters for the third generation and the gaugino sector. For our phenomenological analysis, we simply assume that such a hierarchy occurs for SSB parameters renormalized at a high scale that we take to be $M_{\mathrm{GUT}}$. We also take the less ambitious view that the SUSY flavour and $C P$ problems are ameliorated, but not completely solved, by this hierarchy, and allow the first two generations of scalars to have masses in the $5-20 \mathrm{TeV}$ range, so that some degree of universality/alignment (for the first two generations) is still necessary to satisfy the most stringent flavour constraints. For simplicity, we will assume GUT scale scalar mass universality in the subspace of the first two generations, but allow independent SSB parameters for the third generation and the Higgs sector. This assumption of diagonal GUT scale scalar SSB mass squared matrices undoubtedly has an effect on flavor physics [20], but should have very limited impact on the implications for collider physics and cosmology of the ESUSY scenario - which are after all our main interest in this paper.

The ESUSY model parameter space we will examine is thus given by the set of parameters (renormalized at the GUT scale)

$$
m_{0}(1,2), m_{0}(3), m_{H_{u}}, m_{H_{d}}, A_{0}, m_{1 / 2}, \tan \beta, \operatorname{sign}(\mu)
$$

along with the top quark mass $m_{t}=173.1 \pm 1.3 \mathrm{GeV}$. Here $m_{0}(1,2)$ and $m_{0}(3)$ are the masses of the first/second and of the third generation sfermions, respectively; $m_{H_{u}} \equiv$

\footnotetext{
${ }^{3}$ While the gluino mass parameter can, in principle, be hierarchically larger, RGE effects due to a very heavy gluino would raise third generation squark mass parameters to high values. Gauge coupling unification also prefers that the gluino not be too heavy.
} 
$m_{H_{u}}^{2} / \sqrt{\left|m_{H_{u}}^{2}\right|}$ and $m_{H_{d}} \equiv m_{H_{d}}^{2} / \sqrt{\left|m_{H_{d}}^{2}\right|}$, are the SSB mass parameters of the up- and down-type Higgs scalars; $A_{0}$ is a universal trilinear coupling (relevant mostly only for the third generation); $m_{1 / 2}$ a universal gaugino mass parameter; and $\tan \beta \equiv v_{u} / v_{d}$. Radiative electroweak symmetry breaking can be used to determine $\mu^{2}$ using the measured value of $M_{Z}$.

We wish to maintain the successful gauge coupling unification at $Q=M_{\mathrm{GUT}}$, so that we will assume here that the MSSM is the correct effective field theory between $M_{\mathrm{GUT}}$ and $\widetilde{M}=m_{0}(1,2)$, the scale at which first and second generation scalars decouple from the theory. Below $\widetilde{M}$, we have ESUSY - the SM with two Higgs boson doublets together with third generation scalars, gauginos and higgsinos — as the effective theory.

Models with third generation scalar and gaugino-higgsino sector at $\lesssim \mathrm{TeV}$ but multi$\mathrm{TeV}$ first and second generation mass parameters have been investigated in the past. Shortly after Cohen et al. [9] laid down their framework, it was noted that two loop RGE effects arising due to heavy first/second generation scalars act to suppress third generation scalar mass parameters (even to tachyonic values), and a variety of flavor and $C P$-violating constraints were examined [5, 21, 22]. In refs. [23-25] it was shown that the inverted scalar mass hierarchy which is the hallmark of the ESUSY scenario could emerge dynamically in models with Yukawa coupling unification, although the $\widetilde{M}: M_{\text {weak }}$ ratio was found to be limited $[24,25]$. This class of models - requiring $t-b-\tau$ Yukawa coupling unification and $\mathrm{SO}(10)$ like boundary conditions with $\tan \beta \sim 50-$ has been investigated in detail in refs. [26-35]. In ref. [36], the magnitude of the $\widetilde{M}: M_{\text {weak }}$ hierarchy was investigated in models without Yukawa coupling unification where the matter scalar mass parameters are already taken to be split at the GUT scale. This study assumed equal GUT scale values of third generation and Higgs SSB masses, and as a result, was limited in scope compared to the results to be presented here. Very recently, a dynamical model that leads to first and second generation and heavy Higgs scalars in the $15-20 \mathrm{TeV}$ range, the third generation squarks in the $2-3 \mathrm{TeV}$ range and sub- $\mathrm{TeV}$ electroweak-inos and third generation sleptons has been proposed ref. [37]. In addition, several authors have investigated other related aspects of supersymmetric models with heavy scalars [38-43].

The remainder of this paper is organized as follows. In section 2, we explore the parameter space in eq. (1.2) and map out ranges of parameters that potentially lead to ESUSY at the weak scale. In section 3, we perform a Markov Chain Monte Carlo (MCMC) analysis to search for SUSY scenarios which fulfill the ESUSY conditions, subject to various experimental constraints. We first describe the setup of our MCMC, and then show results for posterior probability distributions both for input parameters that result in ESUSY, as well as for expectations for various sparticle masses and selected experimental observables. Moreover, we pick out some characteristic benchmark points for further study. In section 4, we first present the main particle production rates and decay patterns relevant for LHC phenomenology of the ESUSY scenarios, and then illustrate the diversity of LHC phenomena through more detailed discussion of the phenomenology of the benchmark points, including the possibility that the lightest MSSM sparticle (LMP, as distinct from LSP) may be charged or coloured. We conclude in section 5 with a summary of our results. 


\section{Viable spectra and parameter space of the ESUSY model}

Constrained supersymmetric models such as the mSUGRA model have, for a fixed gluino mass, an upper limit on how massive scalars can be. For a model with universal scalar mass soft terms at the GUT scale, given by $m_{0}$, with $m_{1 / 2}$ fixed, the weak scale value of $\mu^{2}$ diminishes as $m_{0}$ increases. Ultimately, when $m_{0}$ is large enough, $|\mu|$ becomes comparable to the weak scale value of the bino mass $M_{1}$, and one gains neutralinos of mixed higgsinobino composition which make a good candidate for thermal WIMPs. This is the so-called hyperbolic branch/focus point (HB/FP) region of the SUGRA parameter space [44-50]. As $m_{0}$ increases even further, $|\mu|$ decreases even more and the $\widetilde{Z}_{1}$ becomes nearly pure higgsino, and the higgsino-like lightest chargino may drop below limits from LEP2 searches. For yet higher values of $m_{0}, \mu^{2}$ becomes negative, signaling an inappropriate electroweak symmetry breaking (EWSB) pattern. Thus, in the mSUGRA model, for a given value of $m_{1 / 2}, m_{0}$ can become no larger than a few $\mathrm{TeV}$.

If we proceed to models with non-universal generations, taking $m_{0}(1,2)$ as independent of $m_{0}(3)$, with $m_{0}(3)=m_{H_{u}}=m_{H_{d}} \equiv m_{H}$ as in ref. [36], we expect, using 1-loop RGEs, by the same reasoning, an upper bound on $m_{0}(3)$. The point is that the first and second generations essentially decouple from the RG evolution of the Higgs SSB parameters (which enter the electroweak potential minimization conditions, see ref. [51]). At two loop order, however, very large scalar masses do affect the running of the other scalar masses and in fact lead to their suppression at the weak scale [21, 22, 36]. As $m_{0}(3)$ decreases (or $m_{0}(1,2)$ increases $)$, so $m_{0}(3) \ll m_{0}(1,2)$, one of the third generation scalars becomes the LSP, turning tachyonic for even smaller (larger) $m_{0}(3)\left(m_{0}(1,2)\right)$ values. Therefore, with $m_{0}^{2}(3)=m_{H_{u, d}}^{2}$ at the GUT scale, there are both an upper (from $\mu^{2}>0$ ) and a lower (from $\left.m_{\tilde{t}, \tilde{b}, \tilde{\tau}}^{2}>0\right)$ bound on $m_{0}(3)$. In figure 1 , we show regions of allowed parameter space in the $m_{H}$ versus $m_{0}(3)$ plane for $m_{0}(1,2)=5,10$ and $20 \mathrm{TeV}$, with $\left.a\right) m_{1 / 2}=300 \mathrm{GeV}$ and $b$ ) $m_{1 / 2}=800 \mathrm{GeV}$, where the spectrum calculations are performed using Isajet7.80 [5254]. The green dashed line is where $m_{H} \equiv m_{0}(3)$. The region to the right of the blue lines is forbidden due to lack of EWSB, while the region below the red lines is forbidden because a third generation scalar becomes tachyonic. The region to the left and above the contours yields viable spectra.

For low $m_{1 / 2}$, we see from figure $\left.1 a\right)$ that if we restrict our attention to $m_{H}=m_{0}(3)$ (green dashed line) only the interval $1 \mathrm{TeV} \lesssim m_{0}(3)=m_{H} \lesssim 4 \mathrm{TeV}$ is allowed for $m_{0}(1,2)=$ $5 \mathrm{TeV}$, while there are no allowed values for $m_{0}(1,2)=10$ or $20 \mathrm{TeV}$, since for these cases the $m_{0}(3)=m_{H}$ line lies entirely in the excluded region. However, through 1-loop RGE running effects, an increase in $m_{1 / 2}$ increases $m_{\tilde{q}}$. Hence larger values of $m_{0}(1,2)$ become viable if $m_{1 / 2}$ is large enough. This is seen in figure $1 b$ ), where the green dashed line (again representing $m_{0}(3)=m_{H}$ ) shows that, for $m_{1 / 2}=800 \mathrm{GeV}$, the range of allowed $m_{0}(3)=m_{H}$ values is larger for $m_{0}(1,2)=5 \mathrm{TeV}$, where the tachyonic lower bound is gone, so $0<m_{0}(3)=m_{H} \lesssim 5 \mathrm{TeV}$. Besides, if a neutralino LSP is required, we have $0.25<m_{0}(3)=m_{H} \lesssim 5 \mathrm{TeV}$. For the higher $m_{0}(1,2)$ cases we see that the increase in $m_{1 / 2}$ provides an allowed region for $m_{0}(1,2)=10 \mathrm{TeV}$, but still is insufficient for the $m_{0}(1,2)=20 \mathrm{TeV}$ case. It is for this reason that the solutions found in ref. [36] with very 

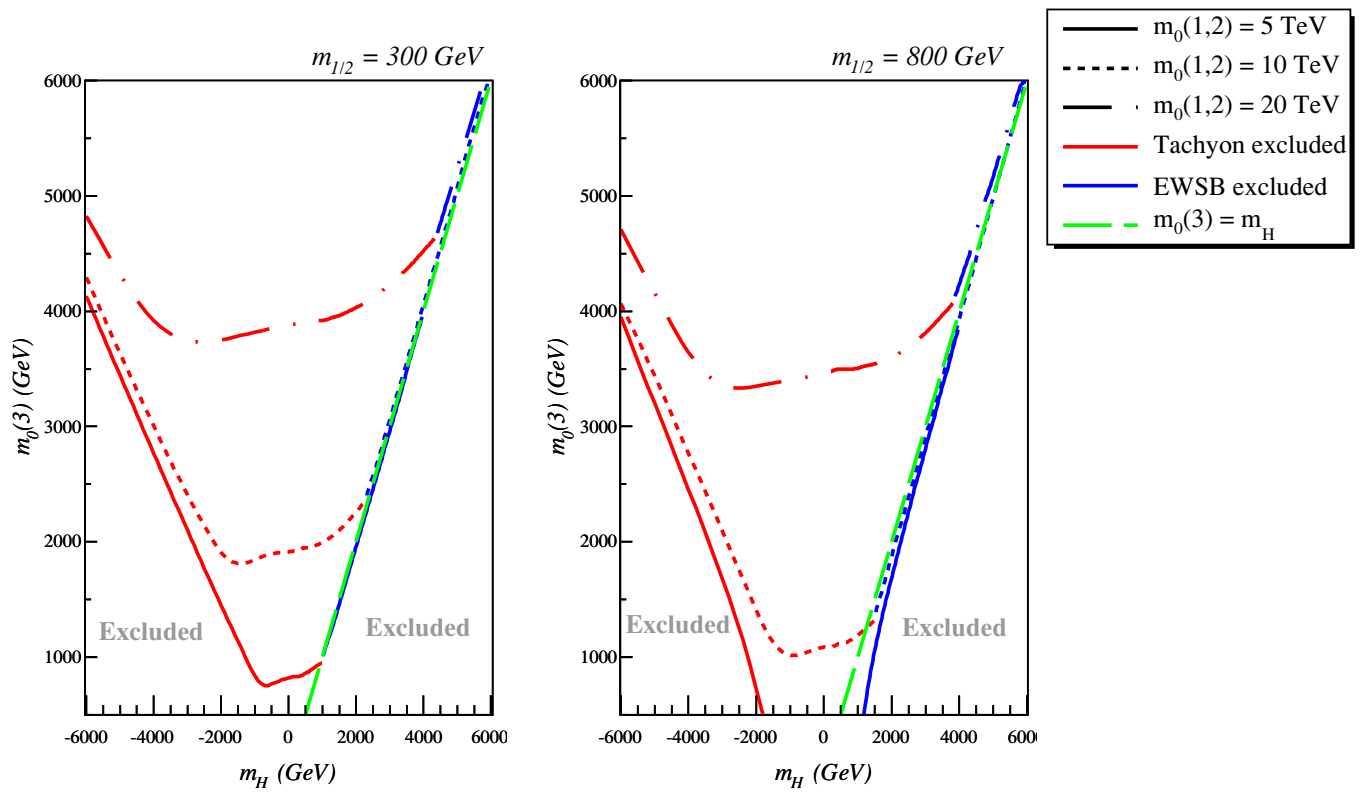

Figure 1. Regions of parameter space leading to viable sparticle mass spectra in the $m_{H} v s . m_{0}(3)$ plane for $m_{0}(1,2)=5,10$ and $20 \mathrm{TeV}$. We fix $A_{0}=0, \tan \beta=10$ and $\mu>0$ and choose $m_{1 / 2}=$ $300 \mathrm{GeV}$ in the left frame and $m_{1 / 2}=800 \mathrm{GeV}$ in the right frame. The region below the red lines is excluded because of tachyonic third generation masses or, for large negative values of $m_{H}$ because $m_{A}^{2}<0$, while the region to the right of the blue lines does not allow EWSB. The green dashed line has $m_{0}(3)=m_{H}$. The ESUSY spectra results from taking $m_{0}(3)$ to its lowest allowed values.

large $m_{0}(1,2)$ values, as needed to suppress FCNCs, also required rather high $m_{1 / 2}$ values, and consequently very heavy gluinos: quite beyond the reach of LHC.

Since there is no compelling theoretical argument to link the Higgs and third generation mass scales, it seems reasonable to adopt independent values of $m_{0}(3)$ and $m_{H_{u}}=m_{H_{d}}=$ $m_{H}$ (NUHM1) or $m_{H_{u}} \neq m_{H_{d}}$ (NUHM2). In this case, for a large value of $m_{0}(3)$, we can take $m_{H}^{2} \ll m_{0}^{2}(3)$, and thus give $m_{H}$ a head start on running towards the necessary negative squared values which are needed for successful EWSB (recall that at the weak scale, $\mu^{2} \simeq-m_{H_{u}}^{2}$ for even modest values of $\tan \beta$ ). This is the well-known feature of NUHM1 models wherein increasing $m_{H}$ results in a decrease of the weak scale value of $|\mu|[55,56]$. Note that large negative values of $m_{H}$ are excluded because there $m_{A}^{2}<0$, signaling that EWSB is not correctly obtained.

If we adopt a low value of $\left|m_{H}\right| \lesssim 1 \mathrm{TeV}$, and then move from high to low $m_{0}(3)$ values, the third generation scalars decrease in mass. The region for ESUSY with third generation scalars at $\lesssim \mathrm{TeV}$ is at $m_{0}(3)$ values just above the tachyonic third generation region. Thus, the strategy for gaining viable ESUSY spectra is to 1.) adopt a large value of $m_{0}(1,2) \sim 5-20 \mathrm{TeV}$, then 2.) adopt a low value of $m_{H} \sim 0-2 \mathrm{TeV}$, and finally, 3.) starting at a several $\mathrm{TeV}$ value of $m_{0}(3)$, decrease its value until third generation scalars dip below the $\mathrm{TeV}$ region. If we want low $|\mu|$ values as well, then increase $m_{H}$ as close to the EWSB boundary (on the right of the figure) as desired.

It is instructive to portray ESUSY in various parameter planes. Figure 2 shows the 


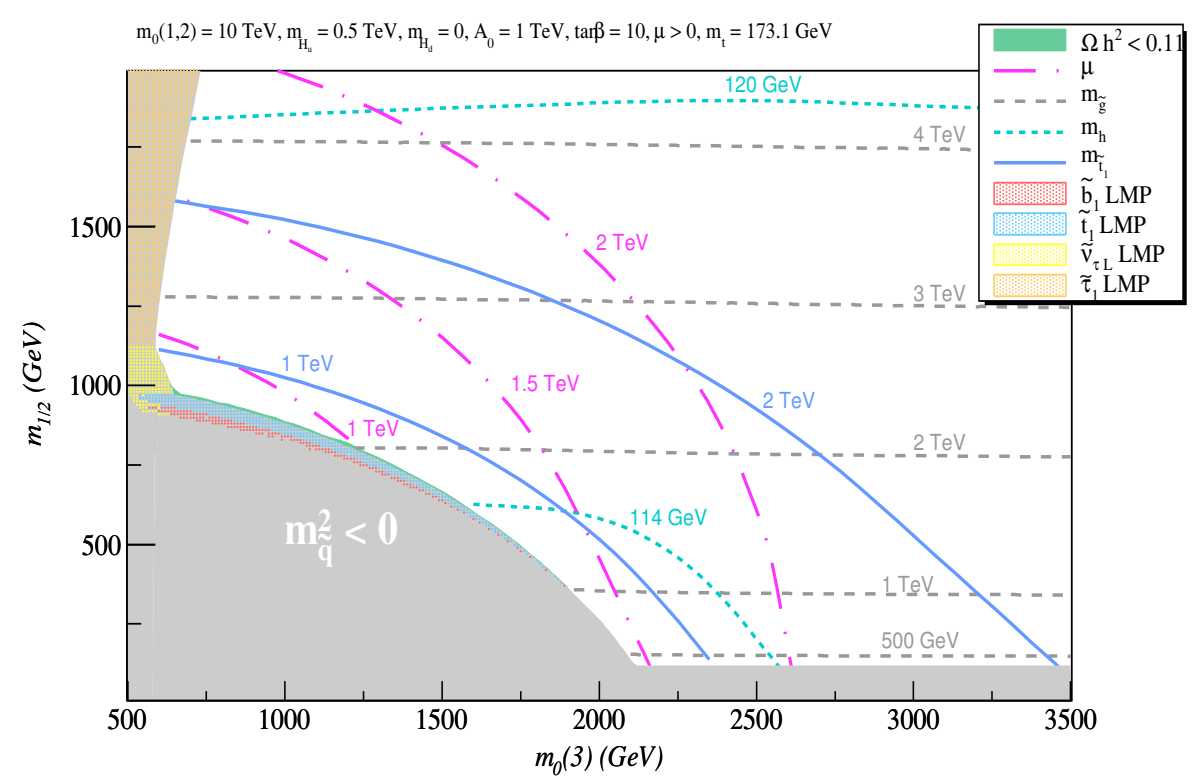

Figure 2. Contours of $\mu$, gluino, stop and Higgs $(h)$ boson masses in the $m_{0}(3) v s . m_{1 / 2}$ plane for $m_{0}(1,2)=10 \mathrm{TeV}, m_{H_{u}}=0.5 \mathrm{TeV}, m_{H_{d}}=0, A_{0}=1 \mathrm{TeV}, \tan \beta=10$ and $\mu>0$. Also shown are regions where various scalars are the lightest MSSM particles (LMPs). In the almost impossible to see narrow green band whose thickness is $\lesssim 10 \mathrm{GeV}$ that hugs the upper boundary of the stop LMP region, the thermal relic abundance $\Omega_{\widetilde{Z}_{1}} h^{2}<0.11$. The gray shaded region is excluded because either we have tachyonic sparticles or the chargino mass is below the corresponding LEP2 limit.

allowed region in the $m_{0}(3)$ vs. $m_{1 / 2}$ plane with $m_{0}(1,2)=10 \mathrm{TeV}, m_{H_{u}}=0.5 \mathrm{TeV}$, $m_{H_{d}}=0 \mathrm{TeV}$ (along with $A_{0}=1 \mathrm{TeV}, \tan \beta=10$ and $\mu>0$ ). The left-side gray shaded region gives tachyonic stop or sbottom masses, while in the lower-right gray region the chargino mass is below its LEP2 limit. The white region gives viable supersymmetric mass spectra. We plot contours of $m_{\tilde{g}}$ (gray dashed), $m_{\tilde{t}_{1}}$ (blue solid), $m_{h}$ (dashed cyan) and $\mu$ (magenta dot-dashed). The region to the lower left of the cyan dashed contour has $m_{h}<114 \mathrm{GeV}$. The slim region adjacent to but right of the tachyonic stop region gives viable ESUSY spectra with top squark masses $m_{\tilde{t}_{1}}<1 \mathrm{TeV}$. There is an almost-impossibleto-see green region of thickness $\lesssim 6 \mathrm{GeV}$ in $m_{1 / 2}$ hugging the boundary of the tachyonic stop region where the thermal neutralino abundance is in accord with measurement: $\Omega_{\widetilde{Z}_{1}} h^{2}<$ 0.11. This is, in fact, the top squark co-annihilation region [57-59] for ESUSY. Along the tachyonic boundary is another narrow region where the $\tilde{t}_{1}$ can become the lightest MSSM particle (LMP), though not necessarily the LSP if a non-MSSM $R$-odd sparticle (such as the axino) is lighter. We also show the regions where $\tilde{b}_{1}, \tilde{\tau}_{1}$ or $\tilde{\nu}_{\tau L}$ is the LMP. ${ }^{4}$

\footnotetext{
${ }^{4}$ With a common value of $m_{0}(3)$, the $\tilde{\nu}_{\tau}$ LMP may be surprising since at the one-loop level Yukawa interactions drive $m_{\tilde{\tau}_{R}}^{2}$ to smaller values than $m_{\tilde{\tau}_{L}}^{2}$. In this case, however, two-loop effects due to heavy scalars are large, and for smaller values of $m_{1 / 2}$ can lead to $m_{\tilde{\tau}_{L}}<m_{\tilde{\tau}_{R}}$ and a sneutrino LMP because of the $D$-term. Since one-loop effects increase with $m_{1 / 2}$, ultimately the usual situation with stau LMP is obtained. Incidently, the compensation between the one and two-loop contributions implies that for certain parameters we can have a near equality of the left- and right-stau mass parameters, and so large stau mixing even though the tau Yukawa coupling is not particularly large.
} 

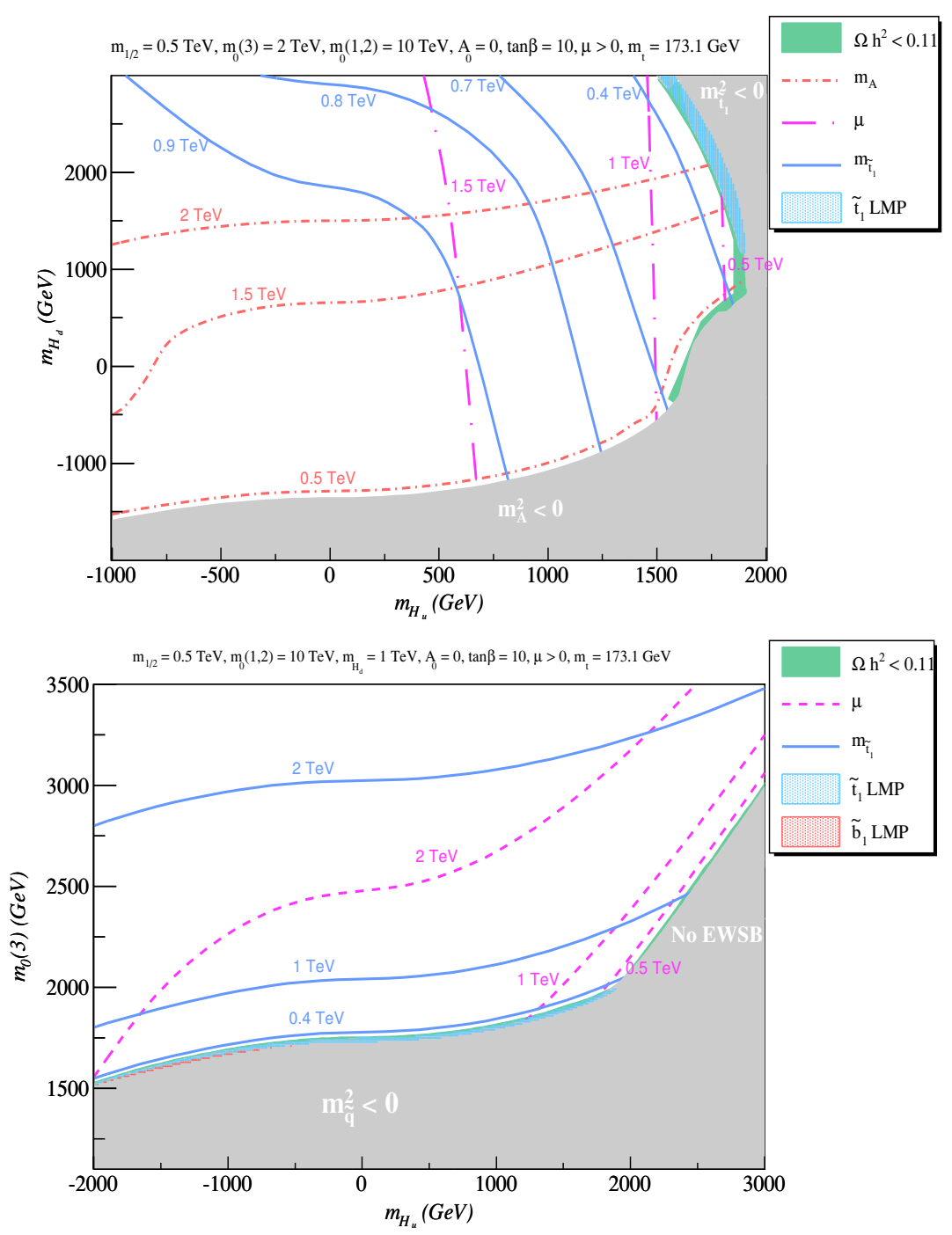

Figure 3. The upper frame a) shows contours of $\mu, m_{\tilde{t}_{1}}$ and $m_{A}$ in the $m_{H_{u}} v s . m_{H_{d}}$ plane for $m_{0}(1,2)=10 \mathrm{TeV}, m_{0}(3)=3 \mathrm{TeV}, m_{1 / 2}=0.5 \mathrm{TeV}, A_{0}=0, \tan \beta=10$ and $\mu>0$. In the green band, the thermal relic abundance $\Omega_{\widetilde{Z}_{1}} h^{2}<0.11$ and in the shaded blue region the $\tilde{t}_{1}$ is the lightest MSSM particle. The gray shaded region is excluded as explained in the text. The lower frame b), shows contours of $\mu$ and $m_{\tilde{t}_{1}}$ in the $m_{H_{u}}$ vs. $m_{0}(3)$ plane for $m_{0}(1,2)=10 \mathrm{TeV}, m_{1 / 2}=0.5 \mathrm{TeV}$, $m_{H_{d}}=1 \mathrm{TeV}, A_{0}=0, \tan \beta=10$ and $\mu>0$.

In figure $3 a$ ), we fix instead the values of $m_{0}(1,2), m_{0}(3)$ and $m_{1 / 2}$ and plot contours of $m_{\tilde{t}_{1}}, \mu$ and $m_{A}$ in the $m_{H_{u}}$ vs. $m_{H_{d}}$ plane. As discussed above, for intermediate positive ranges of $m_{H_{d}}$ in the plot, where $m_{H_{u}} \sim m_{H_{d}}$, as $m_{H_{u}}$ increases, the value of $\mu$ decreases until $\mu^{2}<0$ and EWSB is no longer realized. This can be seen by the $\mu$ contours in this part of the figure where we also see a significant green region where the thermal neutralino relic density is consistent with its observed value. In the lower portion (low $m_{H_{d}}$ ) of the grey area on the right and at the bottom in figure $3 a$ ), we do not obtain radiative EWSB because $m_{h}^{2}$ or $m_{A}^{2}$ become negative. The green region in this vicinity is where the neutralino relic 
density is compatible because of higgs resonance annihilation [60-66]. Finally, for large enough positive values of $m_{H_{u}}$ and $m_{H_{d}}, \tilde{t}_{1}$ becomes the LMP (shaded blue region), with $m_{\tilde{t}}^{2}<0$ above that (upper portion of the grey area on the right) and co-annihilation with stops leads to a compatible neutralino relic density. Finally, we present in figure $3 b$ ) the allowed region in the $m_{H_{u}}$ vs. $m_{0}(3)$ plane with $m_{0}(1,2)=10 \mathrm{TeV}, m_{1 / 2}=0.5 \mathrm{TeV}$ and $m_{H_{d}}=1 \mathrm{TeV}$. We see that the ESUSY scenario is realized at low $m_{0}(3)$ and $m_{H_{u}} \lesssim m_{0}(3)$.

\section{Markov chain Monte Carlo analysis of ESUSY}

In this section, we perform an MCMC analysis to map out the regions of ESUSY parameter space that are consistent with current experimental constraints from collider and flavour physics. The characteristics of ESUSY are enforced by means of theoretical priors. We first briefly describe our set up and list the likelihood functions and model priors that we use, and then show our results for the posterior probability distributions both for input parameters as well as various sparticle masses and selected experimental observables. In section 3.3, we present some benchmark points for more detailed study.

\subsection{Setup of the MCMC scans}

The setup and procedure of our MCMC closely follow [67], so we do not repeat these here but instead just give the key data of the analysis. For details on the MCMC method and Bayesian analysis, see e.g. [68-70]. In addition to Isajet7.80 [52-54], which we use for the spectrum calculation, we use SuperIso2.7 [71] for computing B-physics observables and micrOMEGAs2.4 [72] for the calculation of the neutralino LMP abundance.

For each point $P$ in the scan of ESUSY parameter space, we first compute individual likelihoods $L\left(O_{i}\right)$ for each experimental observable $O_{i}$. Then the overall likelihood is given as the product of these individual likelihoods as $L_{P}=\prod L\left(O_{i}\right)$. The observables we use are listed in table 1 along with the parameters of the corresponding likelihood functions used. The forms of these likelihood functions are given by,

$$
L_{1}\left(x, x_{0}, \sigma_{x}\right)=\frac{1}{1+\exp \left[-\left(x-x_{0}\right) / \sigma_{x}\right]}, \quad L_{2}\left(x, x_{0}, \sigma_{x}\right)=\exp \left[-\frac{\left(x-x_{0}\right)^{2}}{2 \sigma_{x}^{2}}\right],
$$

for observables for which there is only an upper or lower bound, and for observables for which a measurement is available, respectively. For the LEP limit on the Higgs mass, we use [73]

$$
\chi^{2}\left(m_{h}\right)=\frac{\left(m_{h}-m_{h}^{\text {limit }}\right)^{2}}{(1.1 \mathrm{GeV})^{2}+(1.5 \mathrm{GeV})^{2}}
$$

with $m_{h}^{\text {limit }}=115 \mathrm{GeV}$. The likelihood $L\left(m_{h}\right)$ is then given by $L\left(m_{h}\right)=e^{-\chi^{2}\left(m_{h}\right) / 2}$ for $m_{h}<115 \mathrm{GeV}$ and $L\left(m_{h}\right)=1$ for $m_{h} \geq 115 \mathrm{GeV}$.

In order to favour (sub) TeV-scale electroweak gauginos, higgsinos, and third generation sfermions, we multiply the likelihood obtained from the experimental constraints with the following model prior:

$$
L_{M_{\mathrm{eff}}}\left(m_{X}\right)=\frac{1}{1+\exp \left(\left(m_{X}-M_{\mathrm{eff}}\right) / 170 \mathrm{GeV}\right)}
$$




\begin{tabular}{|l|c|l|l|}
\hline Observable & Limit & Likelihood function & ref. \\
\hline $\mathrm{BF}(b \rightarrow s \gamma)$ & $(3.52 \pm 0.34) \times 10^{-4}$ & $L_{2}\left(x, 3.52 \times 10^{-4}, 0.34 \times 10^{-4}\right)$ & {$[74,75]$} \\
\hline $\mathrm{BF}\left(B_{s} \rightarrow \mu^{+} \mu^{-}\right)$ & $\leq 5.8 \times 10^{-8}$ & $L_{1}\left(x, 5.8 \times 10^{-8},-5.8 \times 10^{-10}\right)$ & {$[76]$} \\
\hline$R\left(B_{u} \rightarrow \tau \nu_{\tau}\right)$ & $1.28 \pm 0.38$ & $L_{2}(x, 1.28,0.38)$ & {$[74]$} \\
\hline$m_{t}$ & $173.1 \pm 1.3$ & $L_{2}(x, 173.1,1.3)$ & {$[77]$} \\
\hline$m_{h}$ & $\geq 114.5$ & 1 or $\exp \left(-\chi^{2}\left(m_{h}\right) / 2\right)$ & {$[73]$} \\
\hline SUSY mass limits & LEP limits & 1 or $10^{-9}$ & {$[78]$} \\
\hline
\end{tabular}

Table 1. Observables used in the likelihood calculation.

for each $X=\tilde{\chi}_{1}^{+}, \tilde{\chi}_{2}^{+}, \tilde{t}_{1}, \tilde{t}_{2}, \tilde{b}_{1}$. Choosing, for instance, $M_{\text {eff }}=1.5 \mathrm{TeV}$, we get $L_{M_{\mathrm{eff}}}=$ $0.95,0.5,0.05$ for $m_{X}=1,1.5$ and $2 \mathrm{TeV}$ respectively; for $M_{\text {eff }}=1 \mathrm{TeV}$, we get $L_{M_{\mathrm{eff}}}=$ $0.95,0.5,0.05$ for $m_{X}=0.5,1,1.5 \mathrm{TeV}$ respectively. We have run chains for different $M_{\text {eff }}$ and checked that the effect is indeed only to vary the upper bound on the effective SUSY masses; the qualitative features of the parameter space remain unchanged.

Finally, regarding the model parameters, we allow $m_{1 / 2}=[0,2] \mathrm{TeV}, m_{0}(3)=$ $[0,10] \mathrm{TeV}, m_{H_{u, d}}=[-10,10] \mathrm{TeV}$ (with $m_{H_{u, d}}$ unrelated), $A_{0}=[-40,40] \mathrm{TeV}$, and $\tan \beta=[2,60]$, taking flat prior probability distributions for all these parameters. In addition we let the top mass vary within $m_{t}=173.1 \pm 1.3 \mathrm{GeV}[79]$ with a Gaussian distribution. For $m_{0}(1,2)$, we show results for two different approaches:

1. we let $m_{0}(1,2)$ vary from 5 to $20 \mathrm{TeV}$ with a flat prior probability distribution $\left(L_{\widetilde{M}} \equiv 1\right)$, or

2. we let $m_{0}(1,2)$ vary without limits but apply a model prior of

$$
L_{\widetilde{M}}\left(m_{0}(1,2)\right)=\frac{1}{1+\exp \left(\left(10-m_{0}(1,2)\right) / 1.7 \mathrm{TeV}\right)},
$$

in order to favour $m_{0}(1,2)$ values beyond $10 \mathrm{TeV}$.

The total likelihood of a point is then taken to be $L_{\text {tot }}=L_{P} \times L_{M_{\mathrm{eff}}} \times L_{\widetilde{M}}$.

Before turning to the results, a comment is in order regarding the nature of the LMP. If we do not impose any dark matter requirement on the LMP, the large majority of points have a neutralino LMP with a far too high relic density of up to $\Omega_{\chi} h^{2} \sim 10^{3}$. As noted in the Introduction, this can be in agreement with cosmological observations if the "true" LSP is actually an axino, i.e. in the case of mixed axion/axino dark matter [17-19, 80]. Moreover, about $12 \%$ (4\%) of the points accepted by the chains with $M_{\text {eff }}=1(1.5) \mathrm{TeV}$ have a stop, sbottom or stau LMP. Again these points may be viable if the true LSP is the axino. However, as we will discuss below, the phenomenology in these charged LMP cases is quite different from the neutralino LMP case: in particular, if the stop, sbottom or stau LMP does not decay promptly but gives a charged track. In what follows, we will confine our MCMC analysis to the case of a neutralino LMP, which indeed occurs most frequently. We will, however, comment on the phenomenology of the colored or charged LMP case at the end of the next section. 

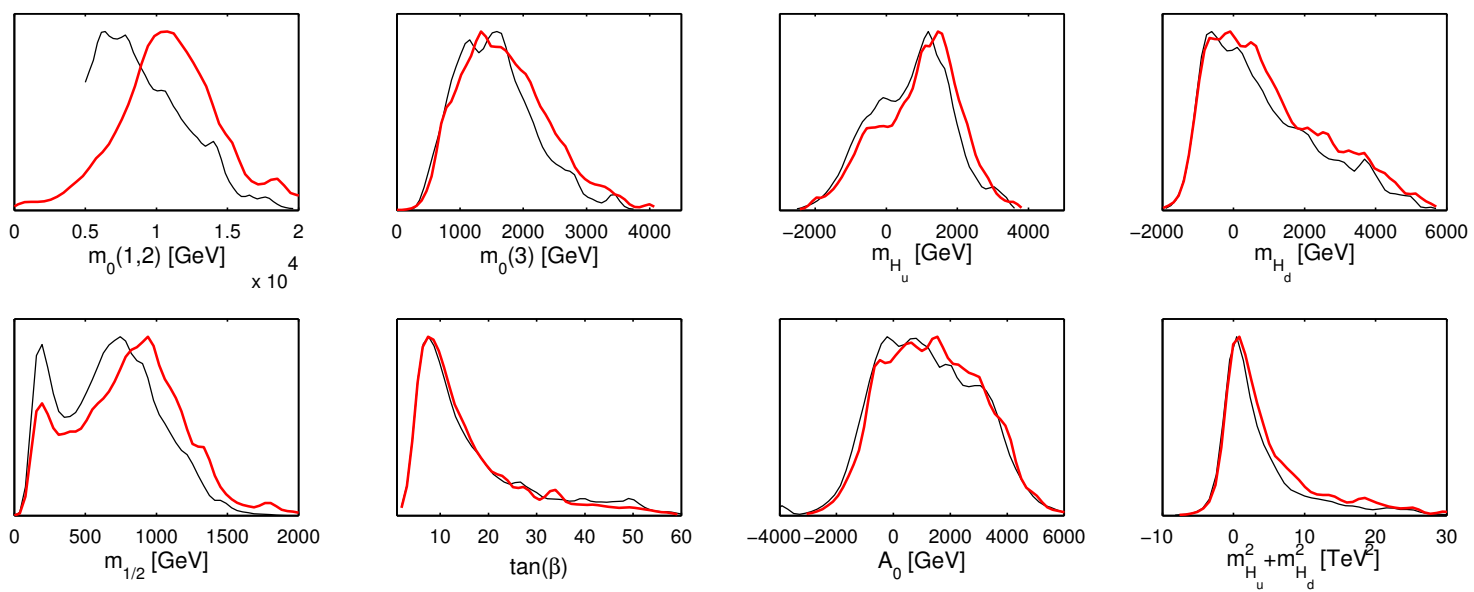

Figure 4. Posterior probability distributions of the input parameters of the ESUSY model from MCMC scans with $M_{\text {eff }}=1 \mathrm{TeV}$, comparing the two priors for $m_{0}(1,2)$. We take $\mu>0$, and assume that the neutralino is the LMP. The thin black lines are for case 1 . where $m_{0}(1,2)=5-20 \mathrm{TeV}$ with a flat distribution, while the thick red lines are for case 2 . which has no limit on $m_{0}(1,2)$ but the prior eq. (3.4) disfavours low values for this parameter. The last panel shows the combination $m_{H_{u}}^{2}+m_{H_{d}}^{2}$.

\subsection{MCMC results with neutralino LMP}

Figure 4 shows the posterior probability distributions of the input parameters of the ESUSY model from MCMC scans requiring a neutralino LMP. Here we have used $M_{\text {eff }}=1 \mathrm{TeV}$ in eq. (3.3). Unseen dimensions are marginalized over. The figure compares the two priors for $m_{0}(1,2)$ : The thin black lines are for case 1 . where $m_{0}(1,2)=5-20 \mathrm{TeV}$ with a flat distribution, while the thick red lines are for case 2 . which has no limits on $m_{0}(1,2)$ but the choice of the prior in eq. (3.4) favours higher values of $m_{0}(1,2)$. We see that, as anticipated, we find solutions where third generation sfermion and gaugino SSB parameters are typically 1-2 TeV, while the first/second generation SSB parameters are favoured to lie beyond $10 \mathrm{TeV}$. Moderate values of $\tan \beta$ are favoured.

As can be seen, the precise condition on $m_{0}(1,2)$ hardly influences the probability distributions of the other parameters. A possible exception is $m_{1 / 2}$, which features a double-peak distribution. This comes from the fact that at small $m_{1 / 2}$, the parameter space volume is constrained in the directions of the scalar mass parameters, while there is more space in the $\tan \beta$ direction. For large $m_{1 / 2}$, on the other hand, $\tan \beta$ is very much constrained by $\mathrm{BR}(b \rightarrow s \gamma)$, while there is more volume in the large $m_{0}(1,2)$ directions. The effect is more pronounced for case 1 . which does not disfavour smaller values for $m_{0}(1,2)$. Moreover, we see that larger $m_{0}(1,2)$ prefers somewhat larger $m_{1 / 2} \cdot{ }^{5}$ It is also interesting to note that very high $m_{0}(1,2)$ around $15-20 \mathrm{TeV}$ suffers from a low probability. This is because as $m_{0}(1,2)$ increases, we are forced to increasing larger values of $m_{0}(3)$ (see

\footnotetext{
${ }^{5}$ The shape of the posterior probability distribution of $m_{1 / 2}$, and $\tan \beta$ are likely the most sensitive to the $b \rightarrow s \gamma$ constraint, and hence to our assumption that there are no off-diagonal pieces in the GUT scale squark mass matrices.
} 

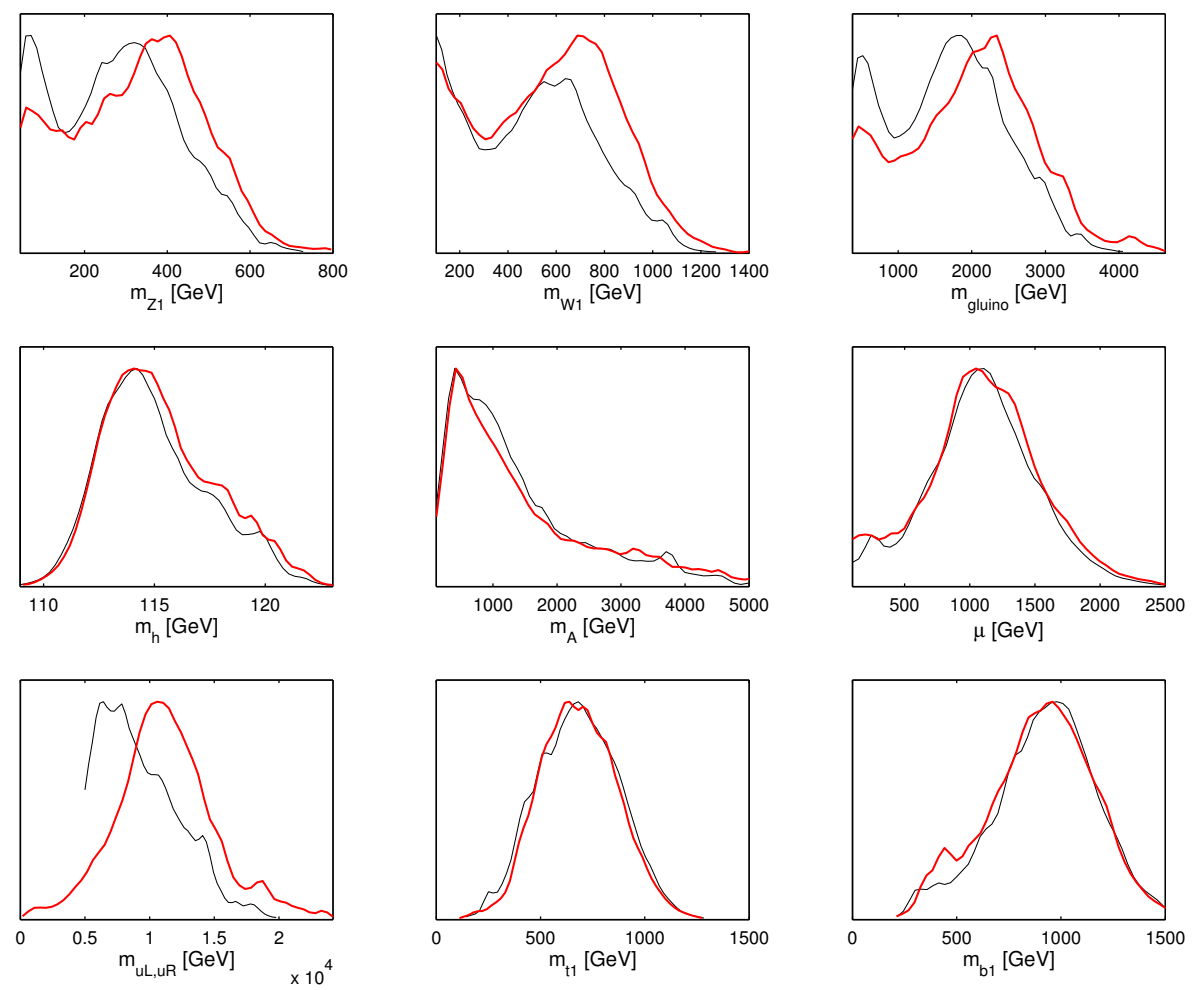

Figure 5. Same as figure 4 but for various sparticle and Higgs masses, and $\mu$.

figure 1). For values of $m_{0}(3)$ in the multi-TeV range, third generation SSB parameters at $\lesssim \mathrm{TeV}$ can occur only when the large two loop RGE contributions rather precisely cancel the naturally multi-TeV contribution that we start with - too little cancellations leave large SSB parameters, while too much cancellation leads to tachyonic masses. As a result, the region of parameter space with a light third generation rapidly shrinks when $m_{0}(1,2)$ exceeds $\sim 15-20 \mathrm{TeV}$.

The posterior probability distributions of several relevant masses are shown in figure 5 . We infer from the EW gaugino spectrum (and also the $\mu$ ) distribution that most of the time the lightest neutralino is bino-like.

Figure 6 displays the probability distributions of $B$-decay observables, $\Delta a_{\mu}=(g-$ $2)_{\mu}^{\mathrm{SUSY}}$, the higgsino fraction $f_{H}=v_{1}^{(1) 2}+v_{2}^{(1) 2}$ (in the notation of ref. [51]) of the $\tilde{Z}_{1}$, and the relic abundance $\Omega h^{2}$ of the $\tilde{Z}_{1}$. It is clear that if the muon anomalous moment is confirmed, it cannot arise within the ESUSY framework. We note that there is nonnegligible fraction of the parameter space with a higgsino-like LMP. We also see that the neutralino relic density peaks around $\Omega h^{2} \simeq 1-10$ and goes up to $\Omega h^{2} \sim 10^{4}$. Nevertheless, solutions with low values of $\Omega h^{2} \lesssim 0.1$, where the relic density of thermal neutralinos does not yield a universe that is too short-lived, also have non-negligible probability. They occur because of Higgs funnel annihilation, coannihilation with light stops and/or sbottoms (or, less likely, staus), or because of a large LMP higgsino component. 

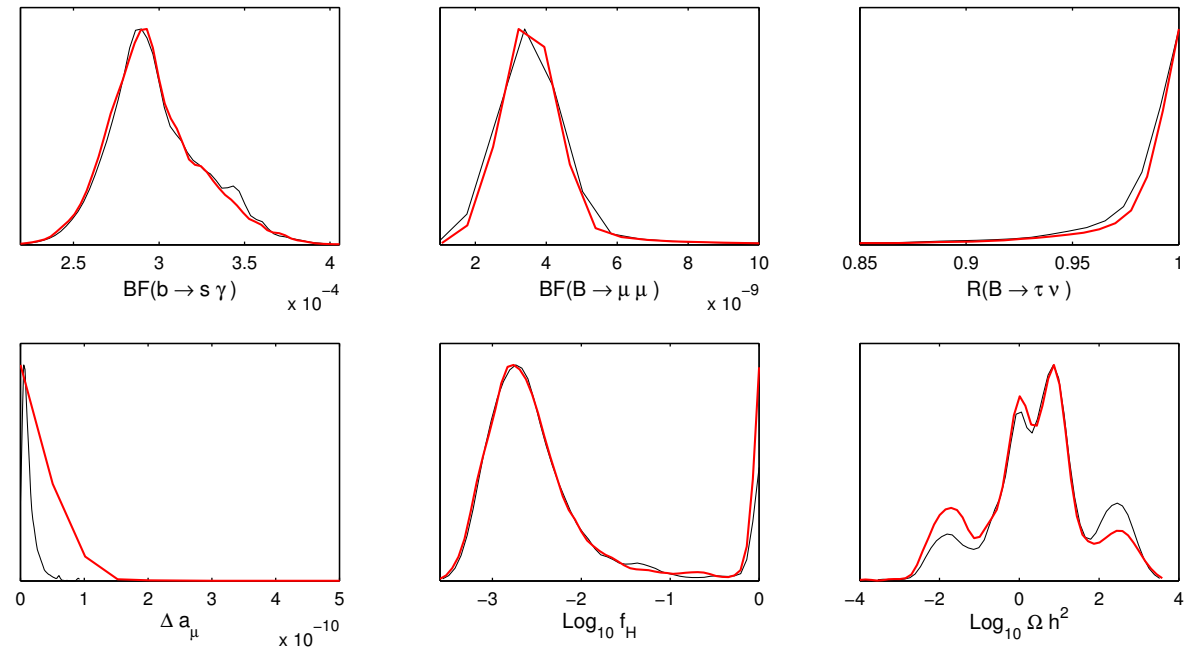

Figure 6. Same as figure 4 but for a variety of low energy observables, the higgsino content of the lightest neutralino, and the thermal neutralino relic density.

\subsection{Effective SUSY benchmark points}

To illustrate the types of SUSY spectra and the diverse phenomenology that can result in ESUSY models, we list five benchmark points in table 2. The first three points we list have a neutralino LMP, while points ES4 and ES5 have a sbottom and a stau LMP, respectively. Point ES1 adopts an $m_{0}(1,2)=10 \mathrm{TeV}$, which is the most favored value in the probability distributions resulting from the MCMC. On the other hand, ES1 has a value of $m_{\tilde{g}}=524 \mathrm{GeV}$, not far from the ESUSY minimum of $m_{\tilde{g}} \gtrsim 460 \mathrm{Gev}$. The chargino $\widetilde{W}_{1}$ and the neutralino $\widetilde{Z}_{2}$ have masses of just $139 \mathrm{GeV}$, and the $\mu$ parameter is $857 \mathrm{GeV}$. All third generation squarks except $\tilde{b}_{2}$, as well as all EW gauginos are $\lesssim 1 \mathrm{TeV}$ scale, while the two staus are over $2 \mathrm{TeV}$.

In point ES2, $m_{0}(1,2)$ is increased to $12 \mathrm{TeV}$. ES2 has much higher value of $m_{\tilde{g}} \sim$ $2.4 \mathrm{TeV}$ with concomitantly heavier $\widetilde{W}_{1}$ and $\widetilde{Z}_{1,2}$, although all EW gauginos along with $\tilde{t}_{1}$, $\tilde{t}_{2}$ and $\tilde{b}_{1}$ are below $1 \mathrm{TeV}$. The $\tilde{b}_{2}$ and staus all have masses $\sim 1.3-1.4 \mathrm{TeV}$ range. For this point, $\tilde{t}_{1}$ decays exclusively via the three-body mode $\tilde{t}_{1} \rightarrow b W \widetilde{Z}_{1}$, while other third generation quarks decay via two-body decays.

Point ES3, with a value of $m_{0}(1,2) \simeq 10 \mathrm{TeV}$, again has all third generation sfermions, as well as all charginos, neutralinos and Higgs bosons essentially at or well below $1 \mathrm{TeV}$. The gluino mass is rather high: $\sim 2.1 \mathrm{TeV}$. One characteristic feature of this point is that, because of the near degeneracy of $\tilde{t}_{1}$ and $\widetilde{Z}_{1}$, not only the two-body decays but even the three body decay $\tilde{t}_{1} \rightarrow b W \widetilde{Z}_{1}$ is kinematically forbidden. In this case, $\tilde{t}_{1}$ will decay via $\tilde{t}_{1} \rightarrow c \widetilde{Z}_{1}$ or $\tilde{t}_{1} \rightarrow b \widetilde{Z}_{1} f \bar{f}^{\prime}$, though of course the small available phase space will favour the former decay [81].

Point ES4 has a high $m_{0}(1,2)=20 \mathrm{TeV}$, a heavy gluino with a mass of $2.5 \mathrm{TeV}$, and correspondingly heavy EW gauginos. However, $\tilde{t}_{1}$ and $\tilde{b}_{1}$ are very light with masses of $327 \mathrm{GeV}$ and $291 \mathrm{GeV}$, while $m\left(\tilde{t}_{2}\right)=793 \mathrm{GeV}$. In this case, $\tilde{b}_{1}$ is the LMP.

Finally, point ES5 has been chosen to illustrate that staus can also be very light. Here, $\tilde{\tau}_{1}$ is the LMP, with a mass of just $289 \mathrm{GeV}$, while $m\left(\tilde{\tau}_{2}\right)=380 \mathrm{GeV}$. The lighter stop and 


\begin{tabular}{|c|c|c|c|c|c|}
\hline parameter & ES1 & ES2 & ES3 & $\mathrm{ES} 4$ & ES5 \\
\hline$m_{0}(1,2)[\mathrm{TeV}]$ & 10 & 12 & 10 & 20 & 6 \\
\hline$m_{0}(3)[\mathrm{TeV}]$ & 2.4 & 1.5 & 1.1 & 3.3 & 0.3 \\
\hline$m_{H_{d}}[\mathrm{TeV}]$ & 1 & -0.35 & -0.6 & 1 & 0 \\
\hline$m_{H_{u}}[\mathrm{TeV}]$ & 2 & 1.2 & 0.5 & 1 & 0 \\
\hline$m_{1 / 2}[\mathrm{TeV}]$ & 0.16 & 1 & 0.85 & 1 & 0.8 \\
\hline$A_{0}[\mathrm{TeV}]$ & 0 & 0.9 & 1 & 0 & 0 \\
\hline$\mu$ & 857 & 902 & 925 & 2329 & 855 \\
\hline$m_{\tilde{g}}$ & 524 & 2446 & 2103 & 2526 & 1941 \\
\hline$m_{\tilde{u}_{L}}[\mathrm{TeV}]$ & 10.0 & 12.1 & 10.1 & 20.1 & 6.2 \\
\hline$m_{\tilde{u}_{R}}[\mathrm{TeV}]$ & 10.0 & 12.2 & 10.1 & 20.1 & 6.2 \\
\hline$m_{\tilde{e}_{L}}[\mathrm{TeV}]$ & 10.0 & 12.0 & 10.0 & 20.0 & 6.0 \\
\hline$m_{\tilde{e}_{R}}[\mathrm{TeV}]$ & 10.0 & 12.0 & 10.0 & 20.0 & 6.0 \\
\hline$m_{\tilde{t}_{1}}$ & 646 & 608 & 398 & 327 & 867 \\
\hline$m_{\tilde{t}_{2}}$ & 1049 & 948 & 770 & 793 & 1147 \\
\hline$m_{\tilde{b}_{1}}$ & 1039 & 830 & 586 & 292 & 1083 \\
\hline$m_{\tilde{b}_{2}}$ & 1711 & 1313 & 958 & 1885 & 1176 \\
\hline$m_{\tilde{\tau}_{1}}$ & 2269 & 1341 & 944 & 2956 & 289 \\
\hline$m_{\tilde{\tau}_{2}}$ & 2299 & 1388 & 1008 & 3152 & 381 \\
\hline$m_{\widetilde{W}_{1}}$ & 139 & 815 & 708 & 881 & 658 \\
\hline$m_{\widetilde{Z}_{2}}$ & 139 & 815 & 708 & 878 & 657 \\
\hline$m_{\widetilde{Z}_{1}}$ & 69 & 441 & 372 & 452 & 347 \\
\hline$m_{A}$ & 1022 & 450 & 398 & 2042 & 875 \\
\hline$m_{h}$ & 110.7 & 118.3 & 117.3 & 119.9 & 117.8 \\
\hline$\Omega_{\widetilde{Z}_{1}} h^{2}$ & 320 & 0.789 & 0.036 & - & - \\
\hline$\sigma[\mathrm{fb}]$ & $23.2 \times 10^{3}$ & 157.8 & 1618.0 & $12.3 \times 10^{3}$ & 51.4 \\
\hline$\tilde{g} \tilde{g}$ & $62.8 \%$ & $0.02 \%$ & $0.01 \%$ & - & $1.5 \%$ \\
\hline$E W-i n o$ pairs & $36.6 \%$ & $5.1 \%$ & $0.8 \%$ & $0.06 \%$ & $35.3 \%$ \\
\hline slep. pairs & - & $0.02 \%$ & $0.01 \%$ & - & $24.4 \%$ \\
\hline$\tilde{t}_{1} \tilde{\tilde{t}}_{1}$ & $0.4 \%$ & $78.3 \%$ & $87.6 \%$ & $28.2 \%$ & $26.5 \%$ \\
\hline$\tilde{t}_{2} \overline{\tilde{t}}_{2}$ & $0.03 \%$ & $4.5 \%$ & $1.8 \%$ & $0.3 \%$ & $3.8 \%$ \\
\hline$\tilde{b}_{1} \overline{\tilde{b}}_{1}$ & $0.02 \%$ & $11.5 \%$ & $9.1 \%$ & $71.4 \%$ & $4.9 \%$ \\
\hline$\tilde{b}_{2} \overline{\tilde{b}}_{2}$ & - & $0.4 \%$ & $0.4 \%$ & - & $2.9 \%$ \\
\hline
\end{tabular}

Table 2. Masses and parameters in GeV units (unless otherwise noted) for five case study points in ESUSY using Isajet 7.80 with $m_{t}=173.1 \mathrm{GeV}, \tan \beta=10$ and $\mu>0$. The LMP mass is denoted with bold numbers. We also list the total tree level sparticle production cross section in $\mathrm{fb}$ at the LHC with a center of mass energy of $14 \mathrm{TeV}$.

the EW gauginos are in the sub- $\mathrm{TeV}$ range, while other third generation sfermions are 1.1-1.2 TeV. 
Two of the ESUSY points with a neutralino as LMP (ES1 and ES2) yield a thermal abundance of neutralinos far in excess of WMAP measured value for the cold DM relic density, while point ES3 is in the top-squark co-annihilation region and gives a thermal neutralino abundance of $\Omega_{\widetilde{Z}_{1}} h^{2}=0.036$. As mentioned previously, the points ES1 and ES2 can still be cosmologically viable if we invoke the PQWW solution to the strong $\mathrm{CP}$ problem, which necessitates the introduction of an axion/axino supermultiplet into the theory. In this case, the axino $\tilde{a}$, and not the neutralino, can be the LSP. If the $\tilde{a}$ is the LSP, then the neutralinos will decay via $\widetilde{Z}_{1} \rightarrow \tilde{a} \gamma$ with lifetime smaller than $\lesssim 0.1-1$ s, i.e. before the onset of Big Bang nucleosynthesis (BBN), for a PQWW scale $f_{a} \lesssim 10^{12} \mathrm{GeV}[82,83]$. Since every neutralino decays to an axino, the would be thermal relic density of neutralinos is reduced by a factor $m_{\tilde{a}} / m_{\widetilde{Z}_{1}}$, and for small enough $m_{\tilde{a}}$ would be compatible with the relic density measurement. Thermal production of axinos in the early universe can still proceed, but the relic abundance of axinos then depends on the re-heat temperature $T_{R}$ after inflation. The dark matter will then consist of an axino/axion admixture [17-19], with relic axions being produced via the vacuum mis-alignment mechanism.

In the cases where $\tilde{t}_{1}, \tilde{b}_{1}$ or $\tilde{\tau}_{1}$ are LMPs, such scenarios are again allowed if, as before, we assume that the axino is the LSP. In these cases the sfermions dominant decay is $\tilde{f}_{1} \rightarrow f \tilde{a}$, via loop-mediated processes; the competing three-body decays, $\tilde{\tau}_{1} \rightarrow \tau \tilde{a} \gamma[84]$ or $\tilde{q} \rightarrow q \tilde{a} g$ [85] are argued to have a small branching fraction. The loop calculation is complicated by the fact that the non-renormalizable axino-bino-photon (and the axinobino-Z and axino-gluino-gluon) coupling enters the calculation. Nevertheless, these authors find that for $m_{\tilde{\tau}}=100 \mathrm{GeV}$, the stau LMP lifetime ranges from about $0.01 \mathrm{~s}$ to $10 \mathrm{~h}$, for the PQWW scale $f_{a}$ in the range $5 \times 10^{9}-5 \times 10^{12} \mathrm{GeV}$, and scales inversely as the stau mass. The corresponding squark lifetime is shorter: about $2 \times 10^{-6} \mathrm{~s}$ for $500 \mathrm{GeV}$ squarks and $1 \mathrm{TeV}$ gluinos with $f_{a}=10^{11} \mathrm{GeV}$. If the LMP lifetime indeed exceeds a few seconds, this could disrupt the successful predictions of Big Bang nucleosynthesis [86], though it appears that if $f_{a}<10^{12} \mathrm{GeV}$, a stau LMP is relatively safe [87]. For a general discussion of charged LMPs, see ref. [88].

\section{Phenomenology of effective SUSY at the LHC}

How will effective SUSY manifest itself at the LHC? To answer this, we first show in figure 7 total gluino and third generation squark pair production cross sections at a $14 \mathrm{TeV} p p$ collider in the $m_{0}(3)$ vs. $m_{1 / 2}$ plane displayed in figure 2 , but now with $m_{0}(1,2)=20 \mathrm{TeV}$. In frame $a$ ), the $p p \rightarrow \tilde{g} \tilde{g} X$ reaction (where $X$ denotes assorted hadronic debris) reaches to over $10^{4} \mathrm{fb}$ for gluino masses as low as $m_{\tilde{g}} \lesssim 500 \mathrm{GeV}$. The gluino pair cross section drops continuously as $m_{1 / 2}$, or alternatively $m_{\tilde{g}}$, increases.

The ESUSY region with third generation squarks having masses $\lesssim \mathrm{TeV}$ lies adjacent to the boundary of the gray tachyonic region. In frame $b$ ), we see the $p p \rightarrow \tilde{t}_{i} \overline{\tilde{t}}_{i}$ plus $\tilde{b}_{i} \overline{\tilde{b}}_{i}$ (for $i=1-2$ ) summed cross sections. These are typically in the 1-100 $\mathrm{fb}$ range all along the tachyonic boundary, with portions reaching cross sections as high as $10^{3} \mathrm{fb}$. Thus, the low $m_{1 / 2}$ region will consist of mainly gluino pair production, where the main influence of light third generation squarks will be upon the gluino branching fractions. As we move to higher 

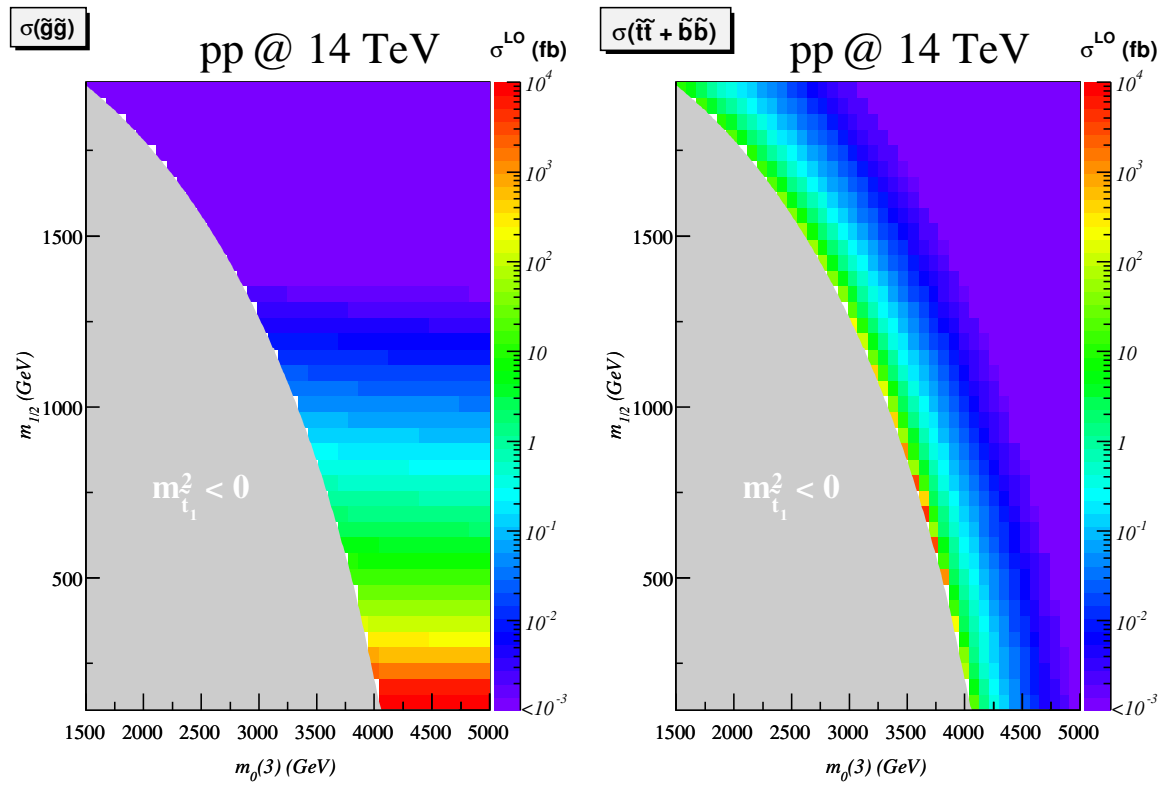

Figure 7. a) Gluino pair production and $b$ ) third generation squark pair production cross sections at LHC with $\sqrt{s}=14 \mathrm{TeV}$ in the $m_{0}(3) v s . m_{1 / 2}$ plane for $m_{0}(1,2)=20 \mathrm{TeV}, m_{H_{u, d}}=1 \mathrm{TeV}$ and $A_{0}=0, \tan \beta=10$ and $\mu>0$.

$m_{1 / 2}$ values, the gluino pair cross section will diminish, and the total SUSY production cross section will be dominated by pair production of third generation squarks.

Unless the gluino is very heavy we would expect that, especially after selection cuts, LHC phenomenology would largely be determined by gluino pair production. SUSY event topologies would thus be sensitive to gluino decay patterns. In figure 8, we show the gluino branching fractions for the MCMC scan points with $m_{\tilde{g}} \leq 1 \mathrm{TeV}$. Of course, when gluino two-body decays are kinematically accessible, these would dominate. We see from the figure that these are accessible for relatively few points when $m_{\tilde{g}}<500 \mathrm{GeV}$. We will return to this later when we discuss how one might distinguish the ESUSY model with light gluinos from the model with $t-b-\tau$ Yukawa unification where $m_{\tilde{g}}$ is bounded from above [26-29]. For the bulk of the scanned points, the gluino decays via three body modes into top and bottom quarks plus EW gauginos; corresponding decays to the first two generations are suppressed because these squarks are very heavy. Bottom-jet tagging will clearly provide an effective way for enriching the ESUSY event sample at the LHC [89-94]. As in many SUSY models, direct decays to the neutralino LMP never have a very large branching fraction. The fact that gluino decays have large branching fractions to $\widetilde{W}_{1}, \widetilde{Z}_{2}$ and top quarks implies that the ESUSY gluino event sample will include multi-lepton events from gluino decay cascades. Finally, we see from the last frame that while the branching fraction for the radiative gluino decays [95] $\tilde{g} \rightarrow g \widetilde{Z}_{i}$ is usually small, it can reach $40-50 \%$. We have checked that the neutralino in question is mostly $\widetilde{Z}_{3,4}$. This occurs when we have large splittings among third generation squarks with stops being lighter than sbottoms, when a light neutralino has a significant up-higgsino component and the decay $\tilde{g} \rightarrow t \bar{t} \widetilde{Z}_{1}$ is 

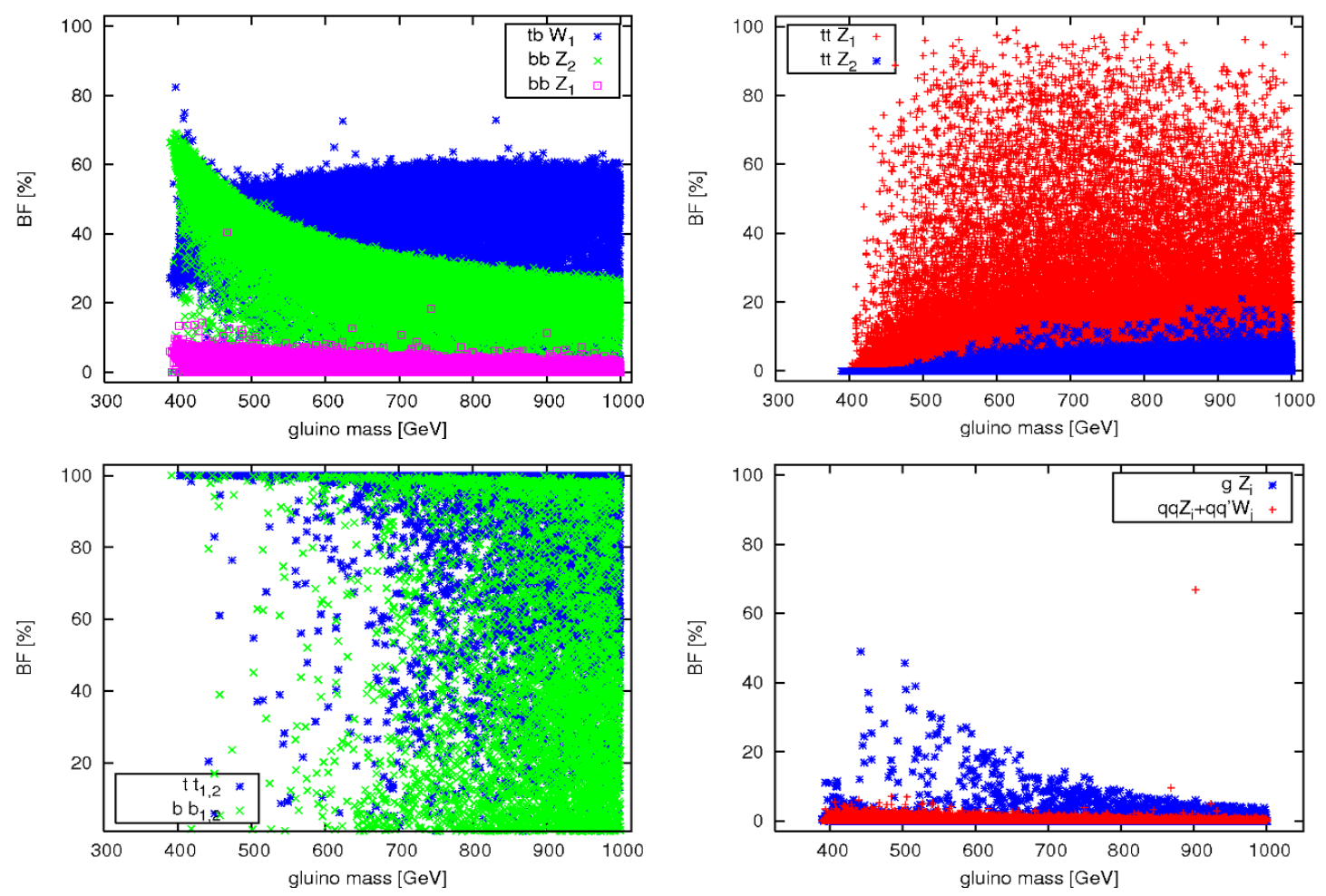

Figure 8. Results for the branching fractions for gluino decays from MCMC scan points with $m_{\tilde{g}} \leq 1 \mathrm{TeV}$ versus the gluino mass. The upper two frames show the branching fractions for three-body decays of gluinos to third generation quarks plus EW gauginos. The bottom-left frame shows the branching fraction for two-body decays to third generation quarks and squarks, while the bottom-right frame illustrates that the radiative decays $\tilde{g} \rightarrow g \widetilde{Z}_{i}$ can sometimes be substantial. All results are for the $L_{\widetilde{M}}=1$ case with $5 \mathrm{TeV}<m_{0}(1,2)<20 \mathrm{TeV}$.

kinematically forbidden. The branching fraction for decays to light quarks is very small for $m_{0}(1,2)>5 \mathrm{TeV}$, although larger values are possible with the second $L_{\widetilde{M}}$ prior (case 2. in section 3.1), which disfavours lower values of $m_{0}(1,2)$ but does not exclude them.

\subsection{Light gluinos with $\widetilde{Z}_{1}$ as LMP (ES1)}

The benchmark point ES1 features a rather light gluino with mass $m_{\tilde{g}}=524 \mathrm{GeV}$, correspondingly light charginos and neutralinos, along with three sub- $\mathrm{TeV}$ third generation squarks. ${ }^{6}$ From table 2, we see that total sparticle production cross section, summed over all reactions, is $23.2 \mathrm{pb}$. Of this, $62.8 \%$ comes from $p p \rightarrow \tilde{g} \tilde{g} X$ production, while $36.6 \%$ comes from EW gaugino production (sum of all chargino and neutralino production cross

\footnotetext{
${ }^{6}$ One might wonder if the Wilson co-efficient $C_{7}\left(M_{W}\right)$ from gluino-squark loops might become large in ESUSY due to broken generations. We have checked using the ISABSG code [96, 97] for model ES1 that we get $C_{7}^{\tilde{g} \tilde{q}}\left(M_{W}\right)=-1.9 \times 10^{-4}$. If instead we adopt generational universality with the same ES1 parameters except $m_{0}(1,2)=3.3 \mathrm{TeV}$, then $C_{7}^{\tilde{g} \tilde{q}}\left(M_{W}\right)$ decreases in magnitude to $-1.4 \times 10^{-4}$. Both these contributions are negligible compared to the SM contribution which yields $C_{7}^{S M}\left(M_{W}\right)=-0.23$.
} 


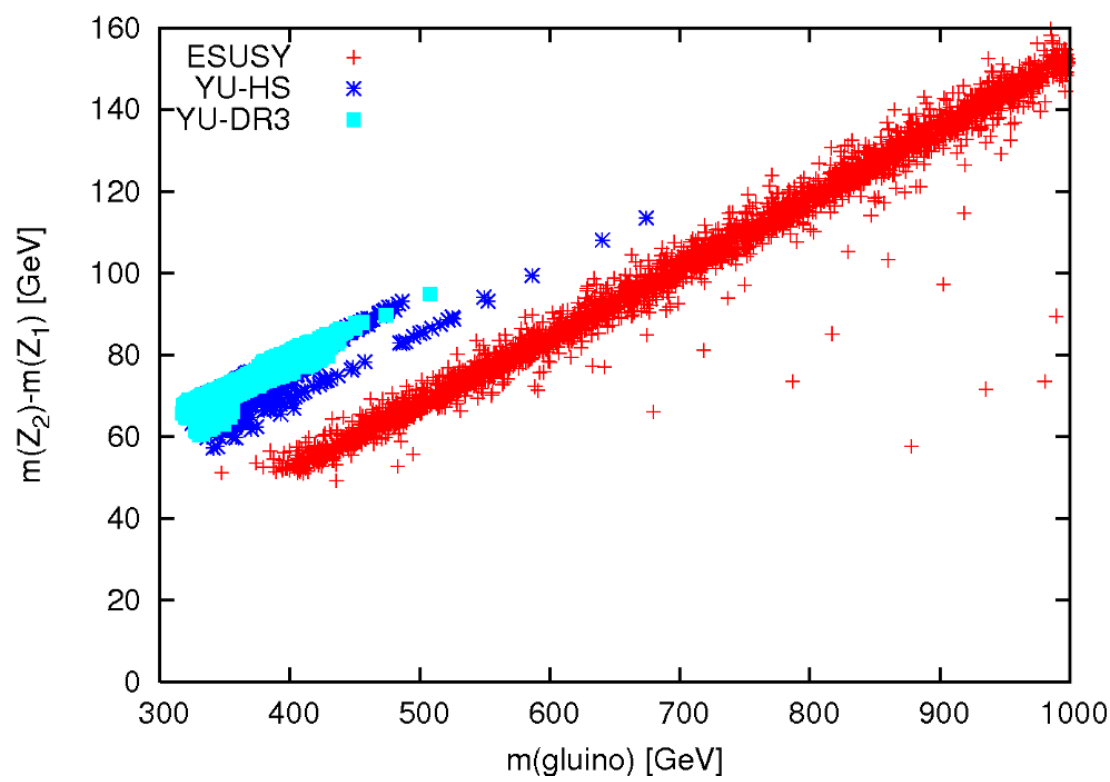

Figure 9. Scatter plot of the gluino mass versus the neutralino mass difference $m_{\widetilde{Z}_{2}}-m_{\widetilde{Z}_{1}}$ that can be obtained from the determination of the dilepton mass edge at the LHC. We show results for the ESUSY model (red), and two different models with $t-b-\tau$ Yukawa unification (medium and light blue) discussed in the text.

sections). Only a tiny fraction of the production cross section comes from third generation squarks, so the rate for $\tilde{t}_{i} \overline{\tilde{t}}_{i}$ and $\tilde{b}_{i} \overline{\tilde{b}}_{i}$ production (for $i=1,2$ ) is very small.

The gluino decays via three body modes as $\tilde{g} \rightarrow b \bar{b} \widetilde{Z}_{2}(37 \%), \tilde{g} \rightarrow t \bar{t} \widetilde{Z}_{1}(19 \%)$ and $\tilde{g} \rightarrow t \bar{b} \widetilde{W}_{1}+c . c(41 \%)$. Thus, each gluino pair production event will contain at least four $b$-jets, plus other jets, isolated leptons (from top quarks and EW gauginos) and $E_{T}^{\text {miss }}$. The $\widetilde{W}_{1}$ decays via three-body modes $\widetilde{W}_{1} \rightarrow \widetilde{Z}_{1} f \bar{f}^{\prime}$ in accord with $W^{*}$ propagator dominance (e.g. $\widetilde{W}_{1} \rightarrow \widetilde{Z}_{1} e \bar{\nu}_{e}$ at $11 \%$ ). The $\widetilde{Z}_{2}$ decays to $b \bar{b} \widetilde{Z}_{1} 20 \%$ of the time, while leptonic decays such as $\widetilde{Z}_{2} \rightarrow \widetilde{Z}_{1} e^{+} e^{-}$occur at the $3 \%$ level. In this case, the $\tilde{b}_{1}$ is quite light and dominantly $\tilde{b}_{L}$, which enhances the $\widetilde{Z}_{2}$ decay to $b$ quarks at the expense of first and second generation fermions. Clean trilepton signals from $\widetilde{W}_{1} \widetilde{Z}_{2}$ production may also be observable [98].

The ES1 scenario will have much the same LHC phenomenology as the Yukawa-unified SUSY with a $500 \mathrm{GeV}$ gluino [93, 94], which is also dominated by gluino pair production followed by three-body gluino decays into mainly b-quarks. A natural question to ask is whether it is possible to distinguish these scenarios at the LHC. One difference is that Yukawa-unified SUSY requires a very large $A_{0}$ parameter with $A_{0} \sim-2 m_{16}$, where $m_{16}$ is the matter GUT scale scalar mass parameter, which lies typically in the range 8-20 TeV. This large value of $A_{0}$ feeds into gaugino mass evolution at two-loops in the RGEs, and suppresses $m_{\tilde{g}}$ with respect to $m_{\widetilde{Z}_{1}}$ and $m_{\widetilde{Z}_{2}, \widetilde{W}_{1}}$.

To illustrate this, we show in figure 9 a scatter plot of $m_{\tilde{g}}$ versus $m_{\widetilde{Z}_{2}}-m_{\widetilde{Z}_{1}}$ obtained by scanning the parameter space of (a) the ESUSY model (red pluses) and (b) the Yukawaunified (YU) model with "just-so" GUT scale Higgs soft mass splitting (HS, dark blue stars) and the YU model with full $D$-term splitting, right-hand neutrinos and 3rd generation 
scalar splitting (the DR3 model [99], light blue squares). Here, we require in YU models that $R$, the ratio of the largest to the smallest of the GUT scale Yukawa couplings, is smaller than 1.05. While there is significant overlap of the two classes of Yukawa-unified models, the ESUSY model lies on a distinct band. We expect that the neutralino mass difference edge will be rather well determined by the $m\left(\ell^{+} \ell^{-}\right)$distribution. Then, even a crude measure of the gluino mass at the 10-20\% level via $M_{\text {eff }}$ [100-102] or total cross section [103] would suffice for this distinction. Also, in ref. [93, 94], it is shown that the mass difference $m_{\tilde{g}}-m_{\widetilde{Z}_{2}}$ might be extracted via the kinematic edge in the $m(b b)$ distribution. ${ }^{7}$ If $m_{\tilde{g}}$ can be determined even more precisely using $m_{T 2}$ [104-108], its generalizations [110-113], kinks [109] or other methods that have recently been suggested, the distinction between the models would be even sharper.

\subsection{Light top and bottom squarks, heavy gluinos and $\widetilde{Z}_{1}$ as LMP (ES2 \& ES3)}

For point ES2, since $m_{1 / 2}$ is large, the value of $m_{\tilde{g}}$ is also large, and so $\tilde{g} \tilde{g}$ will not be produced at an appreciable rate at LHC. Instead, the total SUSY cross section of $\sigma_{S U S Y}=$ $157.8 \mathrm{fb}$ is dominated by $78.3 \% \tilde{t}_{1} \overline{\tilde{t}}_{1}$ production, and $11.5 \% \tilde{b}_{1} \overline{\tilde{b}}_{1}$ production. A few percent of $\tilde{t}_{2} \overline{\tilde{t}}_{2}$ also contribute. The $\tilde{t}_{1} \rightarrow b W \widetilde{Z}_{1}$ decay occurs at nearly $100 \%$ branching fraction, so the $\tilde{t}_{1} \overline{\tilde{t}}_{1}$ production results in a $b \bar{b} W^{+} W^{-}+E_{T}^{\text {miss }}$ final state. This should be separable from $t \bar{t}$ background if large $E_{T}^{\text {miss }}$ or $M_{\text {eff }}$ is required. Another possible background is $Z t \bar{t}$ production. Because gluinos are heavy, SUSY contamination to the signal [92] is not an issue. The $\tilde{b}_{1} \rightarrow W \tilde{t}_{1}$ decays at nearly $100 \%$ branching fraction, so this component of the production cross section will result in a $b \bar{b} W^{+} W^{-} W^{+} W^{-}+E_{T}^{\text {miss }}$ final state.

The case of ES3 has four sub-TeV third generation squarks plus tau-sleptons near a $\mathrm{TeV}$ or below. The total production cross section of $\sigma_{S U S Y}=1618 \mathrm{fb}$ comes from $87.6 \%$ $\tilde{t}_{1} \overline{\tilde{t}}_{1}$ production, $9.1 \% \tilde{b}_{1} \tilde{\tilde{b}}_{1}$ production plus a few percent of heavier top and bottom squark pairs. The visible decay products from $\tilde{t}_{1}$ decay will be soft since there is only a $26 \mathrm{GeV}$ mass gap between the $\tilde{t}_{1}$ and the $\widetilde{Z}_{1}$; the visible decay products of $\tilde{t}_{1}$ may not reliably detectable unless they are boosted. The $\tilde{b}_{1} \rightarrow W^{-} \tilde{t}_{1}$ at nearly $100 \%$ branching fraction, so from $\tilde{b}_{1} \overline{\tilde{b}}_{1}$ production we expect a $W^{+} W^{-}+E_{T}^{\text {miss }}$ final state, accompanied by soft charm jets or other soft hadronic debris. The possible background comes from $W^{+} W^{-} Z$ production.

Before turning to the next benchmark case we remark that, although we have not examined an example of such a benchmark point, it has been shown $[114,115]$ that if the gluinos and stops both have sub-TeV masses and that the decays $\tilde{g} \rightarrow t \tilde{t}_{1} \rightarrow t b \widetilde{W}_{1}$ and/or $\tilde{g} \rightarrow b \tilde{b}_{1} \rightarrow b t \widetilde{W}_{1}$ are kinematically accessible and occur with large branching fractions, a partial reconstruction of the event using techniques to tag the secondary top quark may be possible. Moreover, in [116] it has been shown that if $\tilde{g} \rightarrow t \tilde{t}_{1} \rightarrow t c \widetilde{Z}_{1}$ dominates, the signature of 2 b-jets plus 2 same-sign leptons plus additional jets and missing energy is an excellent discovery channel for gluino masses up to about $900 \mathrm{GeV}$.

\footnotetext{
${ }^{7}$ We have checked that a scatter plot of $m_{\tilde{g}}-m_{\widetilde{Z}_{2}}$ vs, $m_{\widetilde{Z}_{2}}-m_{\widetilde{Z}_{1}}$ also serves to separate the ESUSY model from the Yukawa-unified SUSY scenarios, but do not show it here for brevity.
} 


\subsection{Bottom or top squarks as LMP: the case of heavy quasi-stable colored particles (ES4)}

Benchmark point ES4 illustrates an ESUSY model with $\tilde{t}_{1}$ and $\tilde{b}_{1}$ lighter than $\widetilde{Z}_{1}$, and in fact the bottom squark $\tilde{b}_{1}$ is the LMP. This is viable if the axino is the true LSP so that $\tilde{b}_{1} \rightarrow b \tilde{a}$, and the dark matter consists of an axion/axino admixture. For case ES4, with a PQ breaking scale of order $f_{a} \sim 10^{11} \mathrm{GeV}$, we find a bottom squark lifetime of $\sim 10^{-6}$ sec, i.e. the $\tilde{b}_{1}$ is stable as far as collider searches go, but it decays well before the start of Big Bang nucleosynthesis. Previous searches for quasi-stable squark hadrons $Q$ at ALEPH find that $m_{Q}>95(92) \mathrm{GeV}$ for up-type (down-type) squark hadrons [117]. Searches by CDF evidently require $m_{Q} \gtrsim 241.5 \mathrm{GeV}[118,119]$. At the LHC, for case ES4, sparticles will be produced with total cross section $\sigma_{S U S Y} \simeq 1.2 \times 10^{4} \mathrm{fb}$, of which $71.4 \%$ is $\tilde{b}_{1} \overline{\tilde{b}}_{1}$ production, and $28.2 \%$ is $\tilde{t}_{1} \overline{\tilde{t}}_{1}$ production. The $\tilde{t}_{1} \rightarrow \tilde{b}_{1} W$ branching fraction is essentially $100 \%$, and so augments the $\tilde{b}_{1}$ production rates. The $\tilde{b}_{1}$ is a quasi-stable colored particle, and will hadronize to form an $R$-meson or baryon $B^{ \pm, 0}=\tilde{b}_{1} \bar{q}$ or $\tilde{b}_{1} q q^{\prime}$, where $q$ is usually $u$ or $d$. The properties of squark hadrons have been reviewed in $[119,120]$. The heavy $B$ hadron will be produced and propagate through the detector with minimal energy loss due to hadronic interactions. It can be of charge \pm 1 or 0 , and in fact as it propagates, it has charge exchange reactions with nuclei in the detector material, so that the path of the $B$-hadron can thus be intermittently charged or neutral [121, 122].

Stable squark hadrons can be detected as muon-like events, albeit with the possibility of intermittently appearing and disappearing tracks. Detection can be made using $d E / d x$ measurements in the tracking system, or time-of-flight (ToF) measurements in the muon system. Measuring both the $B$ hadron momentum and velocity should allow a $B$ mass measurement to about 1-2 GeV [119]. The discovery reach of LHC for quasi-stable squark hadrons has been estimated in ref. [119]. Using those results, we estimate the LHC reach with $\sqrt{s}=7 \mathrm{TeV}$ and $1 \mathrm{fb}^{-1}$ of integrated luminosity to be up to $m_{\tilde{b}_{1}} \sim 315 \mathrm{GeV}$, which would already test point ES4! The LHC reach with $\sqrt{s}=14 \mathrm{TeV}$ and $100 \mathrm{fb}^{-1}$ is estimated as $m_{\tilde{b}_{1}} \sim 800 \mathrm{GeV}$, from just $\tilde{b}_{1} \overline{\tilde{b}}_{1}$ production alone. Additional contributions to $\tilde{b}_{1}$ production from cascade decays would increase the reach, and also make clear that $\tilde{b}_{1}$-pair production is not the only new physics process occurring at the LHC.

We note also that many cases with a $\tilde{t}_{1}$ LMP can also be generated in ESUSY. In these cases, with a quasi-stable top-squark hadron, the lifetime and reach discussion is qualitatively similar to that given above.

\subsection{Staus as LMP: the case of heavy quasi-stable charged particles (ES5)}

For ESUSY benchmark point ES5, we have $\tilde{\tau}_{1}$ as LMP. As with point ES4, we may again invoke a lighter axino to escape constraints on charged stable exotics and also mixed axion/axino CDM. In this case, the stau decays through loop diagrams via $\tilde{\tau}_{1} \rightarrow \tau \tilde{a}$ with a lifetime typically of order $1 \mathrm{sec}$ for $f_{a} \sim 10^{11} \mathrm{GeV}$, and does not significantly disrupt BBN [87]. Searches for quasi-stable charged particles at OPAL require $m_{\tilde{\tau}_{1}} \gtrsim 98.5 \mathrm{GeV}$ [123].

The quasi-stable stau will propagate slowly though LHC detectors much like a heavy muon, and leave a highly-ionizing track [121]. The $d E / d x$, ToF and track bending measurements should allow for momentum and velocity, and hence mass determinations. 
For case ES5, the total SUSY production cross section is $\sigma_{S U S Y} \simeq 51.4 \mathrm{fb}$. Of this, just about a quarter comes from stau pair production. However, since all produced sparticles now cascade down into the stau LMP, the total $\tilde{\tau}_{1}$ production is augmented, in this case, by a factor four. For instance, $\tilde{t}_{1} \overline{\tilde{t}}_{1}$ is produced at $26.5 \%$ rate, and $\tilde{t}_{1} \rightarrow \widetilde{Z}_{1} t$ at $53.3 \%$, $\tilde{t}_{1} \rightarrow t \widetilde{Z}_{2}$ at $7.4 \%$ and $\tilde{t}_{1} \rightarrow b \widetilde{W}_{1}$ at $39.3 \%$. Then, $\widetilde{Z}_{1} \rightarrow \tau^{ \pm} \tilde{\tau}_{1}^{\mp}$ at $100 \%$ branching fraction. Also, $\widetilde{W}_{1} \rightarrow \tilde{\tau}_{1} \nu_{\tau}$ at $100 \%$, and $\widetilde{Z}_{2} \rightarrow \tau^{ \pm} \tilde{\tau}_{2}^{\mp}$ at $42 \%$ and $\tilde{\nu}_{\tau} \bar{\nu}_{\tau}+$ c.c at $49 \%$, while $\tilde{\nu}_{\tau} \rightarrow \widetilde{Z}_{1} \nu_{\tau}$, followed by further $\widetilde{Z}_{1}$ decay. Thus, we expect the cascade decay events to each contain at least two $\tilde{\tau}_{1}$ tracks, frequently in company of an assortment of $\tau$-jets, together with $b$ quarks and leptons from $W$-bosons. If the energy of the LMP (as measured from the velocity and momentum of the track) can be included, $E_{T}^{\text {miss }}$ in such SUSY events arises only from neutrinos, and so is not especially large.

The LHC reach for quasi-stable staus has been estimated in [119] in the case where only stau pair production contributes. The reach for LHC at $\sqrt{s}=14 \mathrm{TeV}$ with $100 \mathrm{fb}^{-1}$ of integrated luminosity is found to be $m_{\tilde{\tau}_{1}} \sim 250 \mathrm{GeV}$. This is conservative for the present case, since first we must sum over all slepton pair reactions, and then the summed slepton pair production will be augmented by a factor of four from production via cascade decays of heavier sparticles. ${ }^{8}$ Moreover, as in the ES4 case, detection of more complicated events other than just stau pair production will bring home the richness of the new physics being detected.

\section{Summary and conclusions}

Effective SUSY has been suggested as a model for ameliorating the flavour and $C P$ problems that are endemic to generic SUSY models, while maintaining naturalness in the EWSB sector. The general idea is that SUSY states that have large couplings to the Higgs sector - third generation sfermions and EW gauginos - have masses at or below the TeV scale, while first and second generation sparticles that directly affect the most stringently constrained flavour-changing processes are heavy. The typical ESUSY spectrum consists of multi-TeV first and second generation sfermions along with EW gauginos, Higgs bosons and third generation sfermions at or below the TeV scale; gluinos may be as light as $450 \mathrm{GeV}$ or as heavy as several $\mathrm{TeV}$.

In this paper, we have examined the LHC phenomenology of ESUSY models. Toward this end, we set up the ESUSY model with parameters defined at the GUT scale in section 1. For simplicity of incorporating flavour constraints without greatly impacting LHC physics, we have assumed GUT scale degeneracy of the SSB mass parameters of the first two generations, but allowed the corresponding parameters for the third generation and Higgs scalars to be independent.

We have delineated the viable parameter space in section 2. In section 3.1, we have described the MCMC setup that was used for finding the portion of the entire parameter space consistent with various low energy constraints as well as lower limits on sparticle and Higgs boson masses along with theory priors that were used to obtain ESUSY spectra.

\footnotetext{
${ }^{8}$ In ref. [119], the conservative reach is presented for a single species of stau pair production, where $\sigma\left(p p \rightarrow \tilde{\tau}_{1}^{+} \tilde{\tau}_{1}^{-} X\right) \sim 5 \mathrm{fb}$ for $m_{\tilde{\tau}_{1}}=250 \mathrm{GeV}[124]$.
} 
Our results for the favoured ranges of the input parameters, assuming that the LMP is a neutralino (this is true for the bulk of the points), are shown by the posterior probability distributions in figure 4. The corresponding distributions for sparticle masses, illustrated in figure 5, indeed show the expected qualitative features of the ESUSY spectrum: $\lesssim \mathrm{TeV}$ third generation and EW gaugino masses, 10-20 TeV first/second generation squarks (and sleptons, that we did not show), and gluinos ranging from $0.5-4 \mathrm{TeV}$. The posterior probability distributions for various low energy observables and the thermal neutralino relic density are shown in figure 6. While the former generally lie close to their SM values (because of the nature of the ESUSY spectra), the neutralino relic density $\Omega h^{2}$ ranges from below 0.01 to $10^{3}$ with a peak value around $1-10$.

We note that models with large values of $\Omega_{\widetilde{Z}_{1}} h^{2}$ are phenomenologically every bit as viable as models with a thermal neutralino LSP provided the LMP neutralino is not the true LSP, but decays into the LSP before the advent of Big Bang nucleosynthesis. The axino of the axion-axino multiplet which is present in SUSY models with the PQWW solution to the strong $C P$ problem is an excellent example of an LSP that is not the LMP, and indeed models where the measured CDM is comprised of axions and axinos have been studied in the literature. Moreover, if the axino is the LSP, models where the LMP is charged or coloured are also allowed provided again that the LMP decays before nucleosynthesis.

Our main results on the LHC phenomenology of ESUSY models were presented in section 4. The most important sparticle production mechanisms at the LHC are gluino pair production for values of $m_{1 / 2} \lesssim 700 \mathrm{GeV}$, and third generation squark pair production. A very high $b$-jet multiplicity is the hallmark of multi-jet + multilepton $+E_{T}^{\text {miss }}$ events within the ESUSY framework. Gluinos mostly decay via (real or virtual) third generation squarks (see figure 8) to third generation quarks and EW gauginos. While chargino decay branching fractions tend to follow those of the $W$-boson, the branching fraction for neutralinos to $b$ quarks is often enhanced in these scenarios. Third generation squarks frequently also have $b$-quarks as their decay products. Finally, ESUSY events are also often rich in $t$ quarks whose decays contribute to the multi-lepton component of the signal.

We have discussed the phenomenology of five ESUSY benchmark points introduced in section 3.3. Of these, just the point ES3 has a thermal neutralino relic density in accord with the measured cold DM relic density, although here the $\widetilde{Z}_{1}$ would just make up about $1 / 3$ of the DM. For the other points, the LMP (which need not even be a neutralino) must decay into the true LSP (which might be the axino) plus SM particles. If the LMP is a neutralino, this decay typically occurs well after the neutralino has passed through the LHC detectors, and the LHC phenomenology is essentially the same as for a stable neutralino. The LMP may also be a coloured squark or an electrically charged stau, as illustrated by the last two benchmark points that we discuss. Assuming again that the axino is the LSP, the LMP is quasi-stable and typically traverses the detector before it decays. In these cases, the presence of a (possibly intermittent, in the case of squark LMP) track of a slowly moving particle (whose velocity is obtained through timing information), rather than $E_{T}^{\text {miss }}$, will be the hallmark of SUSY events.

To conclude, effective supersymmetry with third generation squarks and EW gauginos at or below the $\mathrm{TeV}$ scale, but $10-20 \mathrm{TeV}$ first/second generation sfermions ameliorates the 
flavour and $C P$ problems that plague many SUSY models. We have examined the LHC phenomenology of relic-density-consistent ESUSY scenarios and shown that it may differ qualitatively from that in the most frequently examined mSUGRA scenario. The ESUSY picture may also be tested in low energy measurements. In particular, if the branching fraction for $B \rightarrow \tau \nu$ decay, and especially the muon magnetic moment show significant deviation from their SM values, the ESUSY picture would be strongly disfavoured.

\section{Acknowledgments}

We thank A. Pukhov for his help in implementing the Isajet - micrOMEGAs interface for SUSY scenarios with non-universal scalars. XT thanks the UW IceCube collaboration for making his visit to the University of Wisconsin where this work was done, possible. We also thank the Galileo Galilei Institute (GGI), Florence, Italy for hospitality at the time of the Workshop on Dark Matter: Its origin, nature and prospects for detection during which many of the ideas presented in this paper came together. This work was funded in part by the US Department of Energy, and by the French ANR project ToolsDMColl, BLAN07-2-194882.

Open Access. This article is distributed under the terms of the Creative Commons Attribution Noncommercial License which permits any noncommercial use, distribution, and reproduction in any medium, provided the original author(s) and source are credited.

\section{References}

[1] S. Dimopoulos and H. Georgi, Softly broken supersymmetry and SU(5), Nucl. Phys. B 193 (1981) 150 [SPIRES].

[2] N. Sakai, Naturalness in supersymmetric guts, Zeit. Phys. C 11 (1981) 153 [SPIRES].

[3] Y. Nir and N. Seiberg, Should squarks be degenerate?, Phys. Lett. B 309 (1993) 337 [hep-ph/9304307] [SPIRES].

[4] M. Dine, A. Kagan and S. Samuel, Naturalness in supersymmetry, or raising the supersymmetry breaking scale, Phys. Lett. B 243 (1990) 250 [SPIRES].

[5] N. Arkani-Hamed and H. Murayama, Can the supersymmetric flavor problem decouple?, Phys. Rev. D 56 (1997) 6733 [hep-ph/9703259] [SPIRES].

[6] M. Drees, $N=1$ supergravity guts with noncanonical kinetic energy terms, Phys. Rev. D 33 (1986) 1468 [SPIRES].

[7] S. Dimopoulos and G.F. Giudice, Naturalness constraints in supersymmetric theories with nonuniversal soft terms, Phys. Lett. B 357 (1995) 573 [hep-ph/9507282] [SPIRES].

[8] A. Pomarol and D. Tomassini, Horizontal symmetries for the supersymmetric flavor problem, Nucl. Phys. B 466 (1996) 3 [hep-ph/9507462] [SPIRES].

[9] A.G. Cohen, D.B. Kaplan and A.E. Nelson, The more minimal supersymmetric standard model, Phys. Lett. B 388 (1996) 588 [hep-ph/9607394] [SPIRES].

[10] T. Teubner, K. Hagiwara, R. Liao, A.D. Martin and D. Nomura, Update of $g-2$ of the muon and $\Delta \alpha$, arXiv: 1001.5401 [SPIRES]. 
[11] N. Jarosik et al., Seven-Year Wilkinson Microwave Anisotropy Probe (WMAP) Observations: Sky maps, systematic errors and basic results, arXiv:1001.4744 [SPIRES].

[12] E. Komatsu et al., Seven-Year Wilkinson Microwave Anisotropy Probe (WMAP) Observations: Cosmological interpretation, arXiv:1001.4538 [SPIRES].

[13] R.D. Peccei and H.R. Quinn, CP conservation in the presence of instantons, Phys. Rev. Lett. 38 (1977) 1440 [SPIRES].

[14] R.D. Peccei and H.R. Quinn, Constraints imposed by CP conservation in the presence of instantons, Phys. Rev. D 16 (1977) 1791 [SPIRES].

[15] S. Weinberg, A new light boson?, Phys. Rev. Lett. 40 (1978) 223 [SPIRES].

[16] F. Wilczek, Problem of strong $p$ and $t$ invariance in the presence of instantons, Phys. Rev. Lett. 40 (1978) 279 [SPIRES].

[17] H. Baer, A.D. Box and H. Summy, Mainly axion cold dark matter in the minimal supergravity model, JHEP 08 (2009) 080 [arXiv: 0906.2595] [SPIRES].

[18] H. Baer and A.D. Box, Fine-tuning favors mixed axion/axino cold dark matter over neutralinos in the minimal supergravity model, Eur. Phys. J. C 68 (2010) 523 [arXiv: 0910.0333] [SPIRES].

[19] H. Baer, A.D. Box and H. Summy, Neutralino versus axion/axino cold dark matter in the 19 parameter SUGRA model, arXiv:1005.2215 [SPIRES].

[20] A.G. Cohen, D.B. Kaplan, F. Lepeintre and A.E. Nelson, B factory physics from effective supersymmetry, Phys. Rev. Lett. 78 (1997) 2300 [hep-ph/9610252] [SPIRES].

[21] K. Agashe and M. Graesser, Supersymmetry breaking and the supersymmetric flavour problem: An analysis of decoupling the first two generation scalars, Phys. Rev. D 59 (1999) 015007 [hep-ph/9801446] [SPIRES].

[22] J. Hisano, K. Kurosawa and Y. Nomura, Natural effective supersymmetry, Nucl. Phys. B 584 (2000) 3 [hep-ph/0002286] [SPIRES].

[23] J.A. Bagger, J.L. Feng, N. Polonsky and R.-J. Zhang, Superheavy supersymmetry from scalar mass A-parameter fixed points, Phys. Lett. B 473 (2000) 264 [hep-ph/9911255] [SPIRES].

[24] H. Baer, P. Mercadante and X. Tata, Calculable sparticle masses with radiatively driven inverted mass hierarchy, Phys. Lett. B 475 (2000) 289 [hep-ph/9912494] [SPIRES].

[25] H. Baer et al., Aspects of supersymmetric models with a radiatively driven inverted mass hierarchy, Phys. Rev. D 64 (2001) 015002 [hep-ph/0102156] [SPIRES].

[26] H. Baer and J. Ferrandis, Supersymmetric SO(10) GUT models with Yukawa unification and a positive mu term, Phys. Rev. Lett. 87 (2001) 211803 [hep-ph/0106352] [SPIRES].

[27] D. Auto et al., Yukawa coupling unification in supersymmetric models, JHEP 06 (2003) 023 [hep-ph/0302155] [SPIRES].

[28] H. Baer, S. Kraml, S. Sekmen and H. Summy, Dark matter allowed scenarios for Yukawa-unified SO(10) SUSY GUTs, JHEP 03 (2008) 056 [arXiv:0801.1831] [SPIRES].

[29] H. Baer, S. Kraml and S. Sekmen, Is 'just-so' Higgs splitting needed for $t-b-\tau$ Yukawa unified SUSY GUTs?, JHEP 09 (2009) 005 [arXiv: 0908.0134] [SPIRES]. 
[30] T. Blazek, R. Dermisek and S. Raby, Predictions for Higgs and SUSY spectra from $\mathrm{SO}(10)$ Yukawa unification with $\mu>0$, Phys. Rev. Lett. 88 (2002) 111804 [hep-ph/0107097] [SPIRES].

[31] T. Blazek, R. Dermisek and S. Raby, Yukawa unification in $\mathrm{SO}(10)$, Phys. Rev. D 65 (2002) 115004 [hep-ph/0201081] [SPIRES].

[32] R. Dermisek, S. Raby, L. Roszkowski and R. Ruiz De Austri, Dark matter and $B_{s} \rightarrow \mu^{+} \mu^{-}$ with minimal SO(10) soft SUSY breaking, JHEP 04 (2003) 037 [hep-ph/0304101] [SPIRES].

[33] R. Dermisek, S. Raby, L. Roszkowski and R. Ruiz de Austri, Dark matter and $B / s \rightarrow \mu^{+} \mu^{-}$ with minimal $\mathrm{SO}(10)$ soft SUSY breaking. II, JHEP 09 (2005) 029 [hep-ph/0507233] [SPIRES].

[34] W. Altmannshofer, D. Guadagnoli, S. Raby and D.M. Straub, SUSY GUTs with Yukawa unification: A Go/no-go study using FCNC processes, Phys. Lett. B 668 (2008) 385 [arXiv:0801.4363] [SPIRES].

[35] D. Guadagnoli, S. Raby and D.M. Straub, Viable and testable SUSY GUTs with Yukawa unification: the case of split trilinears, JHEP 10 (2009) 059 [arXiv: 0907.4709] [SPIRES].

[36] H. Baer, C. Balázs, P. Mercadante, X. Tata and Y. Wang, Viable supersymmetric models with an inverted scalar mass hierarchy at the GUT scale, Phys. Rev. D 63 (2001) 015011 [hep-ph/0008061] [SPIRES].

[37] S. Schäfer-Nameki, C. Tamarit and G. Torroba, A hybrid higgs, arXiv:1005.0841 [SPIRES].

[38] N. Desai and B. Mukhopadhyaya, Signals of supersymmetry with inaccessible first two families at the large hadron collider, Phys. Rev. D 80 (2009) 055019 [arXiv:0901.4883] [SPIRES].

[39] S.-G. Kim, N. Maekawa, K.I. Nagao, M.M. Nojiri and K. Sakurai, LHC signature of supersymmetric models with non-universal sfermion masses, JHEP 10 (2009) 005 [arXiv: 0907 .4234] [SPIRES].

[40] N. Bernal, A. Djouadi and P. Slavich, The MSSM with heavy scalars, JHEP 07 (2007) 016 [arXiv:0705.1496] [SPIRES].

[41] N. Bernal, Dark matter direct detection in the MSSM with heavy scalars, JCAP 08 (2009) 022 [arXiv: 0905.4239] [SPIRES].

[42] R. Barbieri and D. Pappadopulo, S-particles at their naturalness limits, JHEP 10 (2009) 061 [arXiv:0906.4546] [SPIRES].

[43] M.J. White and F. Feroz, MSSM dark matter measurements at the LHC without squarks and sleptons, JHEP 07 (2010) 064 [arXiv: 1002.1922] [SPIRES].

[44] K.L. Chan, U. Chattopadhyay and P. Nath, Naturalness, weak scale supersymmetry and the prospect for the observation of supersymmetry at the Tevatron and at the LHC, Phys. Rev. D 58 (1998) 096004 [hep-ph/9710473] [SPIRES].

[45] J. Feng, K. Matchev and T. Moroi, Multi - TeV scalars are natural in minimal supergravity, Phys. Rev. Lett. 84 (2000) 2322 [hep-ph/9908309] [SPIRES].

[46] J. Feng, K. Matchev and T. Moroi, Focus points and naturalness in supersymmetry, Phys. Rev. D 61 (2000) 075005 [hep-ph/9909334] [SPIRES]. 
[47] H. Baer, C.-h. Chen, F. Paige and X. Tata, Signals for minimal supergravity at the CERN large hadron collider: Multi - jet plus missing energy channel, Phys. Rev. D 52 (1995) 2746 [hep-ph/9503271] [SPIRES].

[48] H. Baer, C.-h. Chen, F. Paige and X. Tata, Signals for minimal supergravity at the CERN large hadron collider. 2: Multi - lepton channels., Phys. Rev. D 53 (1996) 6241 [hep-ph/9512383] [SPIRES].

[49] H. Baer, C.-h. Chen, M. Drees, F. Paige and X. Tata, Probing minimal supergravity at the CERN LHC for large $\tan \beta$, Phys. Rev. D 59 (1999) 055014 [hep-ph/9809223] [SPIRES].

[50] H. Baer, T. Krupovnickas, S. Profumo and P. Ullio, Model independent approach to focus point supersymmetry: From dark matter to collider searches, JHEP 10 (2005) 020 [hep-ph/0507282] [SPIRES].

[51] H. Baer and X. Tata, Weak scale supersymmetry: From superfields to scattering events, Cambridge University Press, Cambridge U.K. (2006).

[52] F.E. Paige, S.D. Protopopescu, H. Baer and X. Tata, ISAJET 7.69: A Monte Carlo event generator for $p p, \bar{p} p$ and $e^{+} e^{-}$reactions, hep-ph/0312045 [SPIRES].

[53] H. Baer, J. Ferrandis, S. Kraml and W. Porod, On the treatment of threshold effects in SUSY spectrum computations, Phys. Rev. D 73 (2006) 015010 [hep-ph/0511123] [SPIRES].

[54] http://www.nhn.ou.edu/ isajet/.

[55] H. Baer, A. Mustafayev, S. Profumo, A. Belyaev and X. Tata, Neutralino cold dark matter in a one parameter extension of the minimal supergravity model,

Phys. Rev. D 71 (2005) 095008 [hep-ph/0412059] [SPIRES];

[56] H. Baer, A. Mustafayev, S. Profumo, A. Belyaev and X. Tata, Direct, indirect and collider detection of neutralino dark matter in SUSY models with non-universal Higgs masses, JHEP 07 (2005) 065 [hep-ph/0504001] [SPIRES].

[57] C. Böhm, A. Djouadi and M. Drees, Light scalar top quarks and supersymmetric dark matter, Phys. Rev. D 62 (2000) 035012 [hep-ph/9911496] [SPIRES].

[58] J.R. Ellis, K.A. Olive and Y. Santoso, Calculations of Neutralino-Stop Coannihilation in the CMSSM, Astropart. Phys. 18 (2003) 395 [hep-ph/0112113] [SPIRES].

[59] J. Edsjö, et al., Accurate relic densities with neutralino, chargino and sfermion coannihilations in mSUGRA, JCAP 04 (2003) 001 [hep-ph/0301106] [SPIRES].

[60] M. Drees and M.M. Nojiri, The Neutralino relic density in minimal $N=1$ supergravity, Phys. Rev. D 47 (1993) 376 [hep-ph/9207234] [SPIRES].

[61] H. Baer and M. Brhlik, Neutralino dark matter in minimal supergravity: direct detection vs. collider searches, Phys. Rev. D 57 (1998) 567 [hep-ph/9706509] [SPIRES].

[62] H. Baer et al., Yukawa unified supersymmetric $\mathrm{SO}(10)$ model: Cosmology, rare decays and collider searches, Phys. Rev. D 63 (2001) 015007 [hep-ph/0005027] [SPIRES].

[63] J.R. Ellis, T. Falk, G. Ganis, K.A. Olive and M. Srednicki, The CMSSM parameter space at large $\tan \beta$, Phys. Lett. B 510 (2001) 236 [hep-ph/0102098] [SPIRES].

[64] L. Roszkowski, R. Ruiz de Austri and T. Nihei, New cosmological and experimental constraints on the CMSSM, JHEP 08 (2001) 024 [hep-ph/0106334] [SPIRES]. 
[65] A. Djouadi, M. Drees and J.L. Kneur, Constraints on the minimal supergravity model and prospects for SUSY particle production at future linear $e^{+} e^{-}$colliders, JHEP 08 (2001) 055 [hep-ph/0107316] [SPIRES].

[66] A. Lahanas and V. Spanos, Implications of the pseudoscalar Higgs boson in determining the neutralino dark matter, Eur. Phys. J. C 23 (2002) 185 [hep-ph/0106345] [SPIRES].

[67] G. Bélanger, F. Boudjema, A. Pukhov and R.K. Singh, Constraining the MSSM with universal gaugino masses and implication for searches at the LHC, JHEP 11 (2009) 026 [arXiv:0906.5048] [SPIRES].

[68] R.R. de Austri, R. Trotta and L. Roszkowski, A Markov chain Monte Carlo analysis of the CMSSM, JHEP 05 (2006) 002 [hep-ph/0602028] [SPIRES].

[69] B.C. Allanach, K. Cranmer, C.G. Lester and A.M. Weber, Natural priors, CMSSM fits and LHC weather forecasts, JHEP 08 (2007) 023 [arXiv:0705.0487] [SPIRES].

[70] R. Trotta, F. Feroz, M.P. Hobson, L. Roszkowski and R. Ruiz de Austri, The impact of priors and observables on parameter inferences in the constrained MSSM, JHEP 12 (2008) 024 [arXiv:0809.3792] [SPIRES].

[71] F. Mahmoudi, SuperIso v2.3: A program for calculating flavor physics observables in supersymmetry, Comput. Phys. Commun. 180 (2009) 1579 [arXiv:0808.3144] [SPIRES].

[72] G. Bélanger, F. Boudjema, A. Pukhov and A. Semenov, MicrOMEGAs2.0: A program to calculate the relic density of dark matter in a generic model, Comput. Phys. Commun. 176 (2007) 367 [hep-ph/0607059] [SPIRES].

[73] O. Buchmueller et al., Likelihood functions for supersymmetric observables in frequentist analyses of the CMSSM and NUHM1, Eur. Phys. J. C 64 (2009) 391 [arXiv:0907.5568] [SPIRES].

[74] Heavy Flavor Averaging Group collaboration, E. Barberio et al., Averages of b-hadron and c-hadron properties at the end of 200\%, arXiv:0808.1297 [SPIRES].

[75] M. Misiak et al., The first estimate of $B\left(\bar{B} \rightarrow X_{s} \gamma\right)$ at $O\left(\alpha_{s}^{2}\right)$, Phys. Rev. Lett. 98 (2007) 022002 [hep-ph/0609232] [SPIRES].

[76] CDF collaboration, T. Aaltonen et al., Search for $B_{s}^{0} \rightarrow \mu^{+} \mu^{-}$and $B_{d}^{0} \rightarrow \mu^{+} \mu^{-}$decays with $2 \mathrm{fb}^{-1}$ of $p \bar{p}$ collisions, Phys. Rev. Lett. 100 (2008) 101802 [arXiv:0712.1708] [SPIRES].

[77] Tevatron Electroweak Working Group collaboration, Combination of CDF and DO results on the mass of the top quark, arXiv:0903.2503 [SPIRES].

[78] ALEPH, DELPHI, L3 AND OPAL collaborations, LEP2 SUSY Working Group, http://lepsusy.web.cern.ch/lepsusy/.

[79] CDF collaboration, T.E.W. Group, Combination of CDF and DO measurements of the single top production cross section, arXiv:0908.2171 [SPIRES].

[80] H. Baer and H. Summy, SO(10) SUSY GUTs, the gravitino problem, non-thermal leptogenesis and axino dark matter, Phys. Lett. B 666 (2008) 5 [arXiv:0803.0510] [SPIRES].

[81] A.D. Box and X. Tata, Threshold and flavour effects in the renormalization group equations of the MSSM II: Dimensionful couplings, Phys. Rev. D 79 (2009) 035004 [arXiv:0810.5765] [SPIRES].

[82] L. Covi, J.E. Kim and L. Roszkowski, Axinos as cold dark matter, Phys. Rev. Lett. 82 (1999) 4180 [hep-ph/9905212] [SPIRES]. 
[83] L. Covi, H.B. Kim, J.E. Kim and L. Roszkowski, Axinos as dark matter, JHEP 05 (2001) 033 [hep-ph/0101009] [SPIRES].

[84] A. Brandenburg, L. Covi, K. Hamaguchi, L. Roszkowski and F.D. Steffen, Signatures of axinos and gravitinos at colliders, Phys. Lett. B 617 (2005) 99 [hep-ph/0501287] [SPIRES].

[85] L. Covi, L. Roszkowski and M. Small, Effects of squark processes on the axino CDM abundance, JHEP 07 (2002) 023 [hep-ph/0206119] [SPIRES].

[86] K. Jedamzik, Big bang nucleosynthesis constraints on hadronically and electromagnetically decaying relic neutral particles, Phys. Rev. D 74 (2006) 103509 [hep-ph/0604251] [SPIRES].

[87] A. Freitas, F.D. Steffen, N. Tajuddin and D. Wyler, Late energy injection and cosmological constraints in axino dark matter scenarios, Phys. Lett. B 682 (2009) 193 [arXiv:0909.3293] [SPIRES].

[88] C. Berger, L. Covi, S. Kraml and F. Palorini, The number density of a charged relic, JCAP 10 (2008) 005 [arXiv: 0807.0211] [SPIRES].

[89] U. Chattopadhyay, A. Datta, A. Datta, A. Datta and D.P. Roy, LHC signature of the minimal SUGRA model with a large soft scalar mass, Phys. Lett. B 493 (2000) 127 [hep-ph/0008228] [SPIRES].

[90] P.G. Mercadante, J.K. Mizukoshi and X. Tata, Using b-tagging to enhance the SUSY reach of the CERN large hadron collider, Phys. Rev. D 72 (2005) 035009 [hep-ph/0506142] [SPIRES].

[91] S.P. Das, A. Datta, M. Guchait, M. Maity and S. Mukherjee, Focus point SUSY at the LHC revisited, Eur. Phys. J. C 54 (2008) 645 [arXiv:0708.2048] [SPIRES].

[92] R. Kadala J.K. Mizukoshi, P. Mercadante and X. Tata, Heavy-flavour tagging and the supersymmetry reach of the CERN large hadron collider, Eur. Phys. J. 56 (2008) 511 [arXiv: 0803.0001] [SPIRES].

[93] H. Baer, S. Kraml, S. Sekmen and H. Summy, Prospects for Yukawa unified SO(10) SUSY GUTs at the CERN LHC, JHEP 10 (2008) 079 [arXiv:0809. 0710] [SPIRES].

[94] H. Baer, S. Kraml, A. Lessa and S. Sekmen, Testing Yukawa-unified SUSY during year 1 of LHC: the role of multiple b-jets, dileptons and missing $E_{T}, J H E P 02$ (2010) 055 [arXiv: 0911.4739] [SPIRES].

[95] H. Baer, X. Tata and J. Woodside, Phenomenology of gluino decays via loops and top quark Yukawa coupling, Phys. Rev. D 42 (1990) 1568 [SPIRES].

[96] H. Baer and M. Brhlik, QCD improved $b \rightarrow s \gamma$ constraints on the minimal supergravity model, Phys. Rev. D 55 (1997) 3201 [hep-ph/9610224] [SPIRES].

[97] H. Baer, M. Brhlik, D. Castano and X. Tata, $b \rightarrow s \gamma$ constraints on the minimal supergravity model with large $\tan \beta$, Phys. Rev. D 58 (1998) 015007 [hep-ph/9712305] [SPIRES].

[98] H. Baer, C.-h. Chen, F. Paige and X. Tata, Trileptons from chargino - neutralino production at the CERN large hadron collider, Phys. Rev. D 50 (1994) 4508 [hep-ph/9404212] [SPIRES].

[99] H. Baer, S. Kraml and S. Sekmen, Is 'just-so' Higgs splitting needed for $t-b-\tau$ Yukawa unified SUSY GUTs?, JHEP 09 (2009) 005 [arXiv:0908.0134] [SPIRES]. 
[100] I. Hinchliffe, F.E. Paige, M.D. Shapiro, J. Soderqvist and W. Yao, Precision SUSY measurements at CERN LHC, Phys. Rev. D 55 (1997) 5520 [hep-ph/9610544] [SPIRES].

[101] I. Hinchliffe and F.E. Paige, Measurements in gauge mediated SUSY breaking models at CERN LHC, Phys. Rev. D 60 (1999) 095002 [hep-ph/9812233] [SPIRES].

[102] H. Bachacou, I. Hinchliffe and F.E. Paige, Measurements of masses in SUGRA models at CERN LHC, Phys. Rev. D 62 (2000) 015009 [hep-ph/9907518] [SPIRES].

[103] H. Baer, V. Barger, G. Shaughnessy, H. Summy and L.-t. Wang, Precision gluino mass at the LHC in SUSY models with decoupled scalars, Phys. Rev. D 75 (2007) 095010 [hep-ph/0703289] [SPIRES].

[104] C.G. Lester and D.J. Summers, Measuring masses of semiinvisibly decaying particles pair produced at hadron colliders, Phys. Lett. B 463 (1999) 99 [hep-ph/9906349] [SPIRES].

[105] A. Barr, C. Lester and P. Stephens, $m_{T 2}$ : The truth behind the glamour, J. Phys. G 29 (2003) 2343 [hep-ph/0304226] [SPIRES].

[106] W.S. Cho, K. Choi, Y.G. Kim and C.B. Park, Gluino stransverse mass, Phys. Rev. Lett. 100 (2008) 171801 [arXiv:0709. 0288] [SPIRES].

[107] W.S. Cho, K. Choi, Y.G. Kim and C.B. Park, Measuring superparticle masses at hadron collider using the transverse mass kink, JHEP 02 (2008) 035 [arXiv:0711.4526] [SPIRES].

[108] H.-C. Cheng and Z. Han, Minimal kinematic constraints and MT2, JHEP 12 (2008) 063 [arXiv:0810.5178] [SPIRES].

[109] K. Choi, D. Guadagnoli, S.H. Im and C.B. Park, Sparticle masses from transverse mass kinks at the LHC: the case of Yukawa-unified SUSY GUTs, arXiv:1005.0618 [SPIRES].

[110] C. Lester and A. Barr, $m_{T G e n}$ : Mass scale measurements in pair-production at colliders, JHEP 12 (2007) 102 [arXiv:0708.1028] [SPIRES].

[111] B. Gripaios, Transverse observables and mass determination at hadron colliders, JHEP 02 (2008) 053 [arXiv: 0709.2740] [SPIRES].

[112] A.J. Barr, B. Gripaios and C.G. Lester, Transverse masses and kinematic constraints: from the boundary to the crease, JHEP 11 (2009) 096 [arXiv:0908.3779] [SPIRES].

[113] K.T. Matchev, F. Moortgat, L. Pape and M. Park, Precise reconstruction of sparticle masses without ambiguities, JHEP 08 (2009) 104 [arXiv:0906.2417] [SPIRES].

[114] J. Hisano, K. Kawagoe and M.M. Nojiri, A detailed study of the gluino decay into the third generation squarks at the CERN LHC, Phys. Rev. D 68 (2003) 035007 [hep-ph/0304214] [SPIRES].

[115] J. Hisano, K. Kawagoe, R. Kitano and M.M. Nojiri, Scenery from the top: Study of the third generation squarks at CERN LHC, Phys. Rev. D 66 (2002) 115004 [hep-ph/0204078] [SPIRES].

[116] S. Kraml and A.R. Raklev, Same-sign top quarks as signature of light stops at the LHC, Phys. Rev. D 73 (2006) 075002 [hep-ph/0512284] [SPIRES].

[117] ALEPH collaboration, A. Heister et al, Search for stable hadronizing squarks and gluinos in $e^{+} e^{-}$collisions up to $\sqrt{s}=209 \mathrm{GeV}$, Eur. Phys. J. 31 (2003) 327 [hep-ex/0305071] [SPIRES].

[118] CDF collaboration, T. Aaltonen et al., Search for long-lived massive charged particles in $1.96 \mathrm{TeV} \bar{p} p$ collisions, Phys. Rev. Lett. 103 (2009) 021802 [arXiv:0902.1266] [SPIRES]. 
[119] A.R. Raklev, Massive metastable charged (S)particles at the LHC, arXiv:0908.0315 [SPIRES].

[120] M. Fairbairn et al., Stable massive particles at colliders, Phys. Rept. 438 (2007) 1 [hep-ph/0611040] [SPIRES].

[121] M. Drees and X. Tata, Signals for heavy exotics at hadron colliders and supercolliders, Phys. Lett. B 252 (1990) 695 [SPIRES].

[122] H. Baer, K.-m. Cheung and J.F. Gunion, A heavy gluino as the lightest supersymmetric particle, Phys. Rev. D 59 (1999) 075002 [hep-ph/9806361] [SPIRES].

[123] OPAL collaboration, G. Abbiendi et al., Search for stable and long-lived massive charged particles in $e^{+} e^{-}$collisions at $\sqrt{s}=130 \mathrm{GeV}$ to 209-GeV, Phys. Lett. B 572 (2003) 8 [hep-ex/0305031] [SPIRES].

[124] H. Baer, B.W. Harris and M.H. Reno, Next-to-leading order slepton pair production at hadron colliders, Phys. Rev. D 57 (1998) 5871 [hep-ph/9712315] [SPIRES]. 